\title{
Insights into Barley Root Transcriptome under Mild Drought Stress with an Emphasis on Gene Expression Regulatory Mechanisms
}

\author{
Agnieszka Janiak ${ }^{1, *(1)}$, Miroslaw Kwasniewski ${ }^{2}$, Marta Sowa ${ }^{1}$, Anetta Kuczyńska ${ }^{3}$, \\ Krzysztof Mikołajczak ${ }^{3}{ }^{\circledR}$, Piotr Ogrodowicz ${ }^{3}$ and Iwona Szarejko ${ }^{1}$ \\ 1 Institute of Biology, Biotechnology and Environmental Protection, Faculty of Natural Sciences, University of \\ Silesia in Katowice, 40-032 Katowice, Poland; marta.sowa@us.edu.pl (M.S.); iwona.szarejko@us.edu.pl (I.S.) \\ 2 Center of Bioinformatics and Data Analysis, Medical University in Białystok, 15-269 Białystok, Poland; \\ miroslaw.kwasniewski@umb.edu.pl \\ 3 Institute of Plant Genetics, Polish Academy of Sciences, 60-479 Poznań, Poland; akuc@igr.poznan.pl (A.K.); \\ kmik@igr.poznan.pl (K.M.); pogr@igr.poznan.pl (P.O.) \\ * Correspondence: agnieszka.janiak@us.edu.pl; Tel.: +0048-32-2009-457
}

Received: 31 October 2019; Accepted: 3 December 2019; Published: 5 December 2019

\begin{abstract}
Root systems play a pivotal role in coupling with drought stress, which is accompanied with a substantial transcriptome rebuilding in the root tissues. Here, we present the results of global gene expression profiling of roots of two barley genotypes with contrasting abilities to cope with drought that were subjected to a mild level of the stress. We concentrate our analysis on gene expression regulation processes, which allowed the identification of 88 genes from 39 families involved in transcriptional regulation in roots upon mild drought. They include 13 genes encoding transcription factors (TFs) from AP2 family represented by ERFs, DREB, or B3 domain-containing TFs, eight WRKYs, six NACs, five of the HD-domain, MYB or MYB-related, bHLH and bZIP TFs. Also, the representatives of C3H, CPP, GRAS, LOB-domain, TCP, Tiffy, Tubby, and NF-Ys TFs, among others were found to be regulated by the mild drought in barley roots. We found that drought tolerance is accompanied with a lower number of gene expression changes than the amount observed in a susceptible genotype. The better drought acclimation may be related to the activation of transcription factors involved in the maintenance of primary root growth and in the epigenetic control of chromatin and DNA methylation. In addition, our analysis pointed to fives TFs from ERF, LOB, NAC, WRKY and bHLH families that may be important in the mild but not the severe drought response of barley roots.
\end{abstract}

Keywords: barley; drought; roots; stress; transcription factors

\section{Introduction}

Roots are the first organs that perceive the signal of drought stress. Limited water availability has to be sensed by roots, and then the signal is transmitted to the above-ground parts of the plant [1]. At the same time, the root system exhibits large developmental plasticity under abiotic stresses, and understanding the mechanisms that trigger these changes is important to select key factors that are responsible for better plant adaptation to the adverse environmental conditions [2]. The essential role of roots under the abiotic stresses is accompanied by the activation of transcriptome changes, which create a molecular network of responses, and some of its elements may improve plant tolerance to the stress. The spectrum of changes at the molecular level is very broad and includes the alteration of many metabolic pathways, the synthesis of osmoprotectants and antioxidants, or proteins involved in hormone signaling [3,4]. Any transcriptional change relies on the action of transcription factors (TFs) and other proteins that are responsible for gene expression regulation. Factors that are 
differentially expressed under drought stress belong to various TF families, including AP2/ERF, NAC, bZIP, MYB/MYC, bHLH, WRKY, CAMTA, Alfin-like, or homeodomain TFs [5,6] and represent both positive and negative regulators of drought response [7-9]. Specific members of these families have been used to construct overexpression lines that have proven their role in the increase of drought tolerance, either by changes in root growth and architecture [10,11], root anatomy [12], or biochemical composition of root cell walls [13]. The importance of gene expression regulatory mechanisms makes TF encoding genes one of the main targets in the efforts aimed at the development of new varieties better adapted to water scarcity conditions. For this reason, we perform a study of root transcriptomes under water deficit, which we focused mainly on the discovery of gene expression regulatory factors that are involved in the regulation of drought response in this organ. We used barley for this analysis, as it is one of the important cereal species encompassing around $20 \%$ of cereal production in Europe [14]. Also, the availability of the barley genome sequence and its annotation [15] makes it a good model to study gene regulatory network activated in response to drought.

Here, we analyzed two barley genotypes: a European cultivar "Maresi" (Mar) and a Syrian breeding line "Cam/B1/CI08887//CI05761" (CamB). Our previous studies have shown that CamB is more drought tolerant than the European cultivar. Its higher drought tolerance was noticed under severe stress conditions, when plants were subjected to 10 days of stress with the soil water content kept near the permanent wilting point [16,17], and also under mild stress of the same duration, which resembled more natural water shortage conditions $[18,19]$. Leaf and roots transcritome profiling of these genotypes under severe drought stress found genes that predispose the plant to better stress survival. They represent factors involved in the regulatory network of gene expression, signaling mechanisms, with significant contribution of hormone signaling pathways and an interplay between ABA, auxin, ethylene, and brassinosteroid homeostasis or genes encoding LIM domain proteins, which may function as osmotic biosensors. In the presented study, we performed a transcriptome analysis of the whole root system and the second leaf of CamB and Maresi genotypes subjected to 10 days of mild drought at the seedling stage. The transcriptomes of leaves served in the presented study as a background to depict which genes show expression changes in an organ-specific manner. Our data indicate that the mild drought results in more changes in the transcriptomes of roots than in the leaves, and more differentially expressed genes (DEGs) are root-specific. In roots of the CamB genotype, a similar number of DEGs were induced or repressed by the stress, whereas a down-regulation of gene expression prevailed in the roots of more drought susceptible Maresi. We identified 88 DEGs that encode transcription factors or gene expression regulators and the majority of them are root-specific. Their probable function in drought response and tolerance is further discussed, pointing to a possible regulatory network downstream of the selected transcription factors.

\section{Results}

\subsection{An Overview of Root and Leaf Transcriptome Changes in Response to Mild Drought Stress}

Treatment of barley seedlings with a mild drought stress allowed the identification of 2087 differentially expressed genes. We observed a comparable number of DEGs in both genotypes. About 1000 DEGs were found in roots, and nearly 800 were observed in leaves of each genotype (Table 1 and Table S1). A similar number of DEGs were up- or down-regulated in roots of the drought-treated CamB genotype, whereas in the roots of Maresi, the number of down-regulated genes prevailed over the up-regulated ones. In leaves, more genes were up-regulated than down-regulated by the stress in both genotypes. 
Table 1. Summary of differentially expressed genes in CamB and Maresi barley genotypes under drought stress in comparison to control conditions.

\begin{tabular}{cccccc}
\hline \multirow{2}{*}{ Genotype } & \multirow{2}{*}{ Organ } & \multicolumn{2}{c}{ No. of Probes } & \multicolumn{2}{c}{ No. of Genes with Known Annotations * } \\
\cline { 3 - 6 } & & Down-RegulationUp-Regulation & Down-Regulation & Up-Regulation \\
\hline CamB & Roots & 792 & 1019 & 519 & 536 \\
\hline CamB & Leaves & 597 & 705 & 354 & 429 \\
\hline Maresi & Roots & 999 & 884 & 634 & 452 \\
\hline Maresi & Leaves & 602 & 717 & 353 & 436 \\
\hline \multicolumn{5}{r}{$p<0.05 ;$ fold change (FC) $\geq 3 .{ }^{*}$ barley high confidence genes. }
\end{tabular}

Hierarchical clustering of all samples showed that the organ (roots or leaves) was the most discriminative factor dividing the samples into two major transcription profile groups. Smaller differences in transcriptomes were related to the growth conditions (control vs. stress), and the genotype was the least discriminative (Figure S1). The results of hierarchical clustering was supplemented with the values of correlation coefficients between microarray data from each combination of genotype, organ and the type of treatment, calculated for all biological replicates (Table S2). When root and leaf transcriptomes were compared, we found that 947 DEGs were root-specific and much less, 653 DEGs, were expressed specifically in leaves. A group of 144 genes were differentially expressed in an organ- and genotype-independent manner and a set of 343 genes formed different combinations of organ-genotype specific expression patterns (Figure 1A). The whole set of identified DEGs was then functionally analyzed using the KEGG orthology assignment and BRITE functional hierarchies. Importantly, only $25 \%$ of barley DEGs were successfully assigned to KEGG orthology groups and were possible to be mapped into KEGG pathways (Table S3). The BRITE functional hierarchies of identified orthologous groups showed that the majority of DEGs encode enzymes of various metabolic pathways. The remaining genes encode proteins involved in cellular transport, signaling, photosynthesis, DNA repair, or macromolecules protection. We also found a subset of genes involved in gene expression regulation, including transcription factors, or mRNA, tRNA, and ribosome biogenesis (Figure S2). Then, we analyzed root and leaf transcriptomes separately, taking into account the direction of gene expression changes. In roots, the highest number of DEGs, altogether 702 genes, were either up- or down-regulated, commonly in both genotypes. Almost 350 DEGs were up- (178 DEGs) or down-regulated (171 DEGs) exclusively in roots of the CamB genotype, and 378 genes were differentially expressed exclusively in Maresi (97 up-regulated and 281 down-regulated DEGs; Figure 1B). Additionally, five genes showed opposite expression changes in both genotypes-down-regulation in Maresi and up-regulation in the CamB genotype. In leaves, we found a similar number of DEGs, around 350, that were present exclusively either in the CamB or Maresi genotypes. More DEGs were commonly up-regulated than down-regulated in both genotypes (Figure 1C), and one gene had the opposite expression pattern-it was down-regulated in CamB and up-regulated in leaves of the Maresi cultivar.

In order to check the reliability of microarray data, ten randomly selected root DEGs were analyzed using the qPCR method (Table S4). In all cases, significant differences in the fold change of gene expression between control and drought treated samples were obtained. Spearman's rank correlation coefficient (Rs) analysis showed a high correlation in a fold change of expression in both types of data $($ Rs $=0.856 ; p<0.001$; Figure S3), which indicates good quality of microarray analysis. 
A

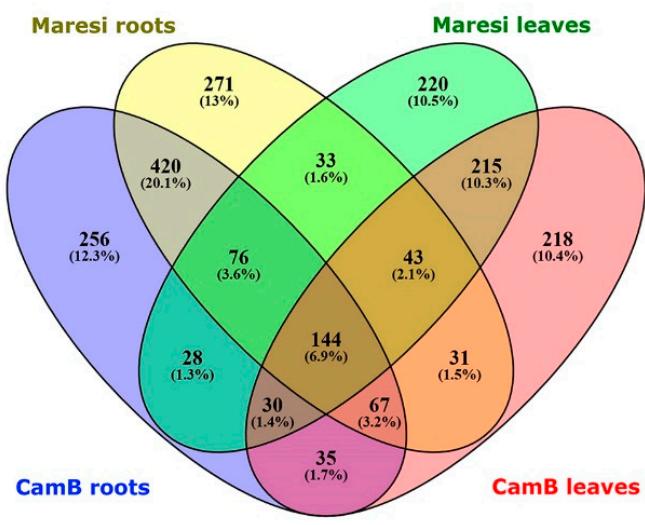

C

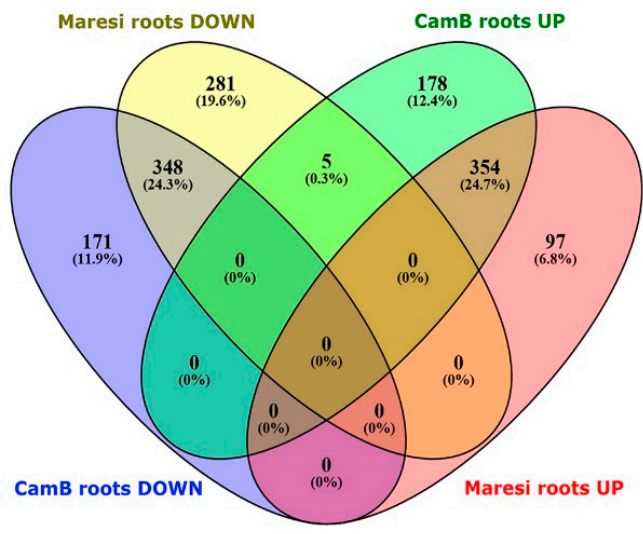

B

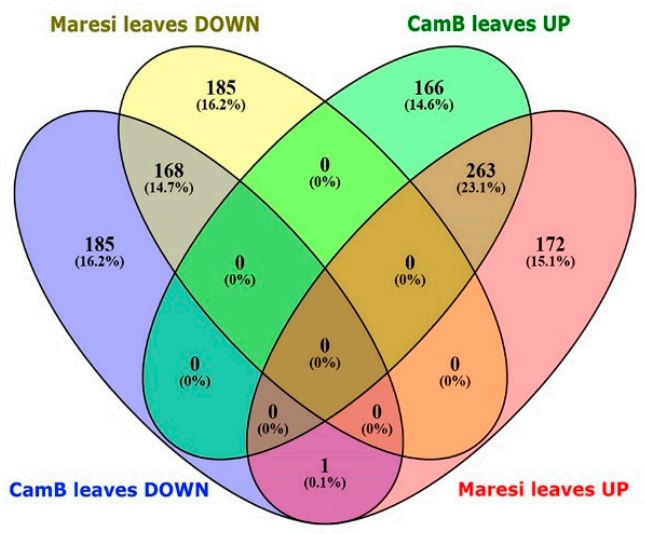

D

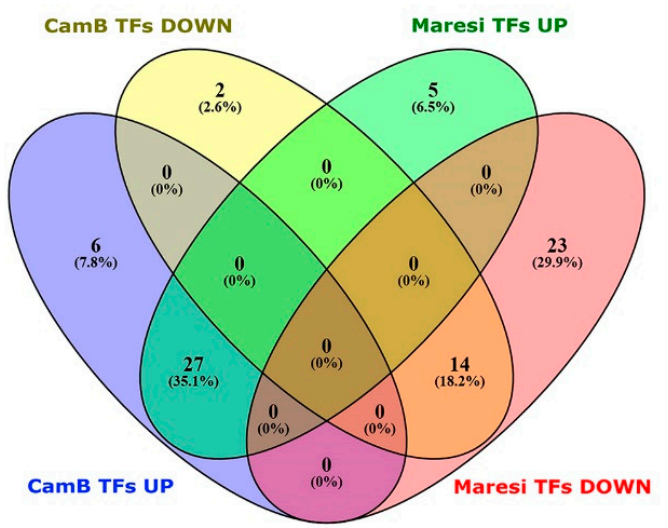

Figure 1. Venn diagrams representing several groups of differentially expressed genes (DEGs). (A) The comparison of a number of DEGs between roots and leaves of CamB and Maresi genotypes. (B) The comparison of a number of up- and down-regulated DEGs in leaves. (C) The comparison of a number of up- and down-regulated DEGs in roots. (D) The comparison of a number of up- and down-regulated DEGs encoding transcription factors and gene expression regulatory proteins identified in the roots of CamB and Maresi.

\subsection{The Characteristics of Root Transcriptomes of Barley Genotypes Exposed to Mild Drought Stress}

Due to a relatively small fraction of genes successfully assigned to KEGG orthology groups, we extended the functional analysis of DEGs detected in roots by gene ontology (GO) enrichment analysis (Table S5), which was later generalized using the GO slim approach. It showed that 10 days of mild drought stress resulted in a significant change of many processes important for root function. The highest number of DEGs belongs to various aspects of biosynthesis processes and the nucleobase-containing compound metabolism, including mostly the regulation of gene expression. Many genes take part in redox processes, cellular transport, protein modification, response to stress and the metabolism of various macromolecules (Figure S4A). Within the majority of these processes, a similar number of genes was either up- or down-regulated, with the exception of protein modifications and carbohydrate metabolism, where more genes were found to be down-regulated. Interestingly, several genes related to photosynthesis process were differentially expressed in roots of both genotypes subjected to drought stress. They represent five genes encoding subunits of photosystem I reaction center, two proteins from photosystem II, plastocyanin, two oxygen-evolving enhancer proteins, and the chlorophyll $a-b$ binding protein. Remarkably, none of these genes were differentially expressed in 
leaves after the 10-days of stress, although we found other genes involved in photosynthesis to be differentially expressed in leaves (Table S6).

Other biological processes that were mostly down-regulated in roots represented reproduction and pollen-pistil interactions, and the majority of genes that were grouped by this GO term encode proteins with serine/threonine kinase activity. The remaining processes affected by the stress were related to translation, signal transduction, cell communication, cell cycle, and cell growth, among others.

The categorization of genes into groups by their molecular function allow us to point to two main categories: binding and several enzymatic activities, represented by oxidoreductases, transferases, hydrolases, or kinases (Figure S4B). Within the binding function, the majority of genes play a role in gene expression regulation, and within the genes encoding kinases, hydrolases, and transferases, many are important in cellular signaling pathways.

The last aspect of gene ontology analysis, cellular localization of proteins encoded by the identified DEGs, showed that their highest number belongs to the membrane proteins, but the important fraction is also localized in the nucleus or extracellular region (Figure S4C).

\subsection{Root DEGs Involved in the Regulation of Gene Expression}

A relatively large group of root DEGs function in the regulation of gene expression. We identified 77 genes encoding various transcription factors belonging to 33 different families, which changed their expression upon the stress in roots. Among these DEGs, eight were differentially expressed specifically in roots of the CamB genotype and 27 were specific to Maresi cultivar, whereas 27 and 14 DEGs were respectively up- or down-regulated in roots of both genotypes (Figure 1D).

About $60 \%$ of all these DEGs were differentially expressed only in roots of at least one genotype, while the remaining genes were also found as DEGs in leaves of either CamB or Maresi (Figure 2, Table S7). The root-specific DEGs encoding TFs belong to 15 different families, and in the majority of cases, these families comprised of one DEG only. The exception is a bHLH TF family that consists of five DEGs and the families that possess a homeodomain coupled with other functional domains, where the other five genes were classified (HB-BELL, HB-HD-ZIP, and HB-KNOX families). An overrepresentation of root-specific DEGs was also found in the bZIP and WRKY families (Figure 2, Table S7).

In addition to the group of DEGs for transcription factors, eleven DEGs were classified as genes encoding other types of transcriptional regulators. They include auxine responsive proteins of the AUX/IAA family, mitochondrial transcription termination factors (mTERF), the PHD finger proteins, tumor necrosis receptor-associated factor (TRAF) family proteins, an ethylene receptor, and a protein with BAH domain. Eight of these DEGs were down-regulated, and nine were root-specific (Table S7).

In order to find the possible regulatory factors that may contribute to the drought tolerance or drought susceptibility, we selected the genes that were differentially expressed in roots in a genotype-specific manner. There were altogether ten DEGs specific to the drought-tolerant CamB genotype, representing eight genes encoding transcription factors-six of them were up-regulated, and seven were differentially expressed only in the roots of this genotype. There were also two genes encoding regulatory proteins, one up- and one down-regulated, and both of them were specific to the roots (Table 2). 


\begin{tabular}{|c|c|c|c|}
\hline \multirow[b]{3}{*}{ Family name } & \multicolumn{2}{|c|}{ Expression change } & \multirow{3}{*}{$\begin{array}{l}\text { No. of root } \\
\text { specific } \\
\text { DEGs }\end{array}$} \\
\hline & UP & DOWN & \\
\hline & $\begin{array}{c}\text { CamB vs Mar } \\
\text { DEGs } \\
\text { distribution }\end{array}$ & $\begin{array}{c}\text { CamB vs Mar } \\
\text { DEGs } \\
\text { distribution }\end{array}$ & \\
\hline AP2/ERF-ERF & 2 & 12 & 3 \\
\hline AP2/ERF-RAV & & 1 & 0 \\
\hline B3 & 1 & 1 & 3 \\
\hline $\mathrm{bHLH}$ & & 2 & 5 \\
\hline bZIP & 0 & 0 & 4 \\
\hline C2C2-CO-like & & 0 & 1 \\
\hline C2C2-GATA & & & 0 \\
\hline $\mathrm{C} 3 \mathrm{H}$ & 0 & & 0 \\
\hline CPP & 0 & & 1 \\
\hline GARP-ARR-B & & 0 & 1 \\
\hline GARP-G2-like & 1 & 0 & 2 \\
\hline General TF & 0 & & 1 \\
\hline GRAS & 0 & 0 & 1 \\
\hline HB-BELL & & 0 & 1 \\
\hline HB-HD-ZIP & 0 & 1 & 2 \\
\hline HB-KNOX & & $\begin{array}{lll}0 & 0 & 2\end{array}$ & 2 \\
\hline HSF & 40 & & 0 \\
\hline
\end{tabular}

\begin{tabular}{|c|c|c|c|}
\hline \multirow{3}{*}{ Family name } & \multicolumn{2}{|c|}{ Expression change } & \multirow{3}{*}{$\begin{array}{c}\text { No. of root } \\
\text { specific } \\
\text { DEGs }\end{array}$} \\
\hline & UP & DOWN & \\
\hline & $\begin{array}{c}\text { CamB vs Mar } \\
\text { DEGs } \\
\text { distribution }\end{array}$ & $\begin{array}{c}\text { CamB vs Mar } \\
\text { DEGs } \\
\text { distribution }\end{array}$ & \\
\hline LOB & & & 1 \\
\hline MADS-M-type & 1 & 2 & 2 \\
\hline MYB & 0 & & 0 \\
\hline MYB-related & & 2 & 1 \\
\hline NAC & 0 & 1 & 1 \\
\hline NF-YA & 0 & & 1 \\
\hline NF-YB & 0 & & 1 \\
\hline RWP-RK & 0 & & 1 \\
\hline S1Fa-like & & & 1 \\
\hline SBP & & 0 & 1 \\
\hline SCAI TF & 0 & & 1 \\
\hline STAT & & 0 & 1 \\
\hline TCP & & 0 & 2 \\
\hline Tify & & 0 & 0 \\
\hline TUB & & 10 & 0 \\
\hline WRKY & 0 & 0 & 6 \\
\hline
\end{tabular}

Figure 2. An overview of the gene number and root-specific differentially expressed genes (DEGs) encoding transcription factors differentially expressed after mild drought treatment in roots of CamB and Maresi genotypes. Cam-CamB genotype, Mar-Maresi genotype. 
Table 2. The list of DEGs involved in the gene expression regulation processes that were specific to the roots of the drought-tolerant CamB genotype.

\begin{tabular}{|c|c|c|c|c|c|c|}
\hline \multirow{2}{*}{ Horvu ID } & \multicolumn{4}{|c|}{ Expression Change } & \multirow{2}{*}{$\begin{array}{c}\text { Family } \\
\text { Name }\end{array}$} & \multirow{2}{*}{ Gene Description } \\
\hline & CamB Roots & $\begin{array}{c}\text { CamB } \\
\text { Leaves }\end{array}$ & $\begin{array}{c}\text { Mar } \\
\text { Roots }\end{array}$ & $\begin{array}{c}\text { Mar } \\
\text { Leaves }\end{array}$ & & \\
\hline \multicolumn{7}{|c|}{ Transcription factors: } \\
\hline HORVU4HR1G087580 & up & $\mathrm{n} / \mathrm{c}$ & $\mathrm{n} / \mathrm{c}$ & $\mathrm{n} / \mathrm{c}$ & bHLH & Uncharacterized \\
\hline HORVU3Hr1G073470 & up & $\mathrm{n} / \mathrm{c}$ & $\mathrm{n} / \mathrm{c}$ & $\mathrm{n} / \mathrm{c}$ & $\mathrm{CPP}$ & $\begin{array}{c}\text { Protein } \\
\text { tesmin/TSO1-like } \\
\text { CXC } 5\end{array}$ \\
\hline HORVU2Hr1G020140 & up & $\mathrm{n} / \mathrm{c}$ & $\mathrm{n} / \mathrm{c}$ & up & GARP-G2-like & $\begin{array}{l}\text { Two-component } \\
\text { response regulator } \\
\text { ARR18 }\end{array}$ \\
\hline HORVU7Hr1G096430 & down & $\mathrm{n} / \mathrm{c}$ & $\mathrm{n} / \mathrm{c}$ & $\mathrm{n} / \mathrm{c}$ & GARP-G2-like & $\begin{array}{c}\text { Myb-like } \\
\text { transcription factor } \\
\text { family protein }\end{array}$ \\
\hline HORVU5Hr1G110960 & up & $\mathrm{n} / \mathrm{c}$ & $\mathrm{n} / \mathrm{c}$ & $\mathrm{n} / \mathrm{c}$ & General TF & $\begin{array}{c}\text { Transcription } \\
\text { initiation factor IIE } \\
\text { subunit beta }\end{array}$ \\
\hline HORVU1Hr1G071620 & up & $\mathrm{n} / \mathrm{c}$ & $\mathrm{n} / \mathrm{c}$ & $\mathrm{n} / \mathrm{c}$ & NF-YB & $\begin{array}{c}\text { Nuclear transcription } \\
\text { factor Y subunit B }\end{array}$ \\
\hline HORVU5Hr1G062040 & up & $\mathrm{n} / \mathrm{c}$ & $\mathrm{n} / \mathrm{c}$ & $\mathrm{n} / \mathrm{c}$ & $\begin{array}{l}\text { SCAI TF } \\
\text { family }\end{array}$ & Protein SCAI \\
\hline HORVU3Hr1G061930 & down & down & $\mathrm{n} / \mathrm{c}$ & down & TUB & $\begin{array}{l}\text { Tubby-like F-box } \\
\text { protein } 1\end{array}$ \\
\hline \multicolumn{7}{|c|}{ Transcriptional Regulators: } \\
\hline HORVU5Hr1G061120 & down & $\mathrm{n} / \mathrm{c}$ & $\mathrm{n} / \mathrm{c}$ & $\mathrm{n} / \mathrm{c}$ & $\begin{array}{c}\text { BAH } \\
\text { domain }\end{array}$ & $\begin{array}{c}\text { Bromo-adjacent } \\
\text { homology (BAH) } \\
\text { domain-containing } \\
\text { protein }\end{array}$ \\
\hline HORVU6Hr1G034680 & up & $\mathrm{n} / \mathrm{c}$ & $\mathrm{n} / \mathrm{c}$ & $\mathrm{n} / \mathrm{c}$ & PHD & $\begin{array}{c}\text { methyl-CPG-binding } \\
\text { domain } 9\end{array}$ \\
\hline
\end{tabular}

A similar analysis was performed for the drought susceptible cultivar Maresi, and here, almost $3 \times$ more DEGs (28 DEGs) encoding transcription factors were found to be specific to this genotype. The majority (23 DEGs) were down-regulated, and the expression of most of them was not affected by the stress in leaves. Among those genes that were differentially expressed also in Maresi leaves, one gene, encoding a protein from the TIFY family, showed an opposite expression change in the two organs. Additionally, three genes encoding transcriptional regulators were also found. Two of them were specific to the roots of Maresi, and one showed an opposite expression pattern in leaves and roots. This gene encodes a putative E3 ubiquitin-protein ligase and was also up-regulated in the leaves of the CamB genotype (Table 3). 
Table 3. The list of DEGs involved in the gene expression regulation processes that were specific to the roots of the drought susceptible Maresi genotype.

\begin{tabular}{|c|c|c|c|c|c|c|}
\hline \multirow[b]{2}{*}{ Horvu ID } & \multicolumn{4}{|c|}{ Expression change } & \multirow[b]{2}{*}{ Family Name } & \multirow{2}{*}{ Gene Description } \\
\hline & CamB Roots & CamB Leaves & Mar Roots & Mar Leaves & & \\
\hline \multicolumn{7}{|c|}{ Transcription factors: } \\
\hline HORVU2Hr1G050260 & $\mathrm{n} / \mathrm{c}$ & $\mathrm{n} / \mathrm{c}$ & up & $\mathrm{n} / \mathrm{c}$ & AP2/ERF-ERF & Ethylene-responsive transcription factor \\
\hline HORVU4Hr1G077310 & $\mathrm{n} / \mathrm{c}$ & $\mathrm{n} / \mathrm{c}$ & down & $\mathrm{n} / \mathrm{c}$ & AP2/ERF-ERF & Ethylene-responsive transcription factor \\
\hline HORVU5Hr1G080300 & $\mathrm{n} / \mathrm{c}$ & $\mathrm{n} / \mathrm{c}$ & down & down & AP2/ERF-ERF & Dehydration-responsive element-binding protein 1B \\
\hline HORVU6Hr1G080340 & $\mathrm{n} / \mathrm{c}$ & up & up & up & AP2/ERF-ERF & Ethylene-responsive transcription factor 5 \\
\hline HORVU3Hr1G105720 & $\mathrm{n} / \mathrm{c}$ & $\mathrm{n} / \mathrm{c}$ & down & $\mathrm{n} / \mathrm{c}$ & B3 & B3 domain-containing protein \\
\hline HORVU5Hr1G017450 & $\mathrm{n} / \mathrm{c}$ & $\mathrm{n} / \mathrm{c}$ & up & $\mathrm{n} / \mathrm{c}$ & B3 & B3 domain-containing protein \\
\hline HORVU3Hr1G108680 & $\mathrm{n} / \mathrm{c}$ & $\mathrm{n} / \mathrm{c}$ & up & $\mathrm{n} / \mathrm{c}$ & bHLH & Transcription factor ORG2 \\
\hline HORVU5Hr1G002090 & $\mathrm{n} / \mathrm{c}$ & $\mathrm{n} / \mathrm{c}$ & down & $\mathrm{n} / \mathrm{c}$ & bHLH & Basic helix-loop-helix (bHLH) DNA-binding superfamily protein \\
\hline HORVU6Hr1G071950 & $\mathrm{n} / \mathrm{c}$ & $\mathrm{n} / \mathrm{c}$ & down & $\mathrm{n} / \mathrm{c}$ & $\mathrm{C} 2 \mathrm{C} 2-\mathrm{CO}-$ like & Zinc finger protein CONSTANS-LIKE 10 \\
\hline HORVU4Hr1G088280 & $\mathrm{n} / \mathrm{c}$ & up & up & up & C2C2-GATA & GATA transcription factor 2 \\
\hline HORVU3Hr1G085720 & $\mathrm{n} / \mathrm{c}$ & $\mathrm{n} / \mathrm{c}$ & down & $\mathrm{n} / \mathrm{c}$ & HB-BELL & BEL1-like homeodomain 8 \\
\hline HORVU5Hr1G067010 & $\mathrm{n} / \mathrm{c}$ & $\mathrm{n} / \mathrm{c}$ & down & $\mathrm{n} / \mathrm{c}$ & HB-HD-ZIP & Homeobox-leucine zipper protein family \\
\hline HORVU2Hr1G061320 & $\mathrm{n} / \mathrm{c}$ & $\mathrm{n} / \mathrm{c}$ & down & $\mathrm{n} / \mathrm{c}$ & HB-KNOX & Homeobox protein knotted-1-like 12 \\
\hline HORVU5Hr1G098570 & $\mathrm{n} / \mathrm{c}$ & $\mathrm{n} / \mathrm{c}$ & down & $\mathrm{n} / \mathrm{c}$ & HB-KNOX & Homeobox protein knotted-1-like 12 \\
\hline HORVU0Hr1G003020 & $\mathrm{n} / \mathrm{c}$ & $\mathrm{n} / \mathrm{c}$ & down & $\mathrm{n} / \mathrm{c}$ & MADS-M-type & MADS-box transcription factor 18 \\
\hline HORVU3Hr1G095090 & $\mathrm{n} / \mathrm{c}$ & $\mathrm{n} / \mathrm{c}$ & down & $\mathrm{n} / \mathrm{c}$ & MADS-M-type & MADS-box transcription factor family protein \\
\hline HORVU3Hr1G052710 & $\mathrm{n} / \mathrm{c}$ & $\mathrm{n} / \mathrm{c}$ & down & $\mathrm{n} / \mathrm{c}$ & MYB-related & Myb-like transcription factor family protein \\
\hline HORVU6Hr1G066000 & $\mathrm{n} / \mathrm{c}$ & down & down & $\mathrm{n} / \mathrm{c}$ & MYB-related & Myb-like transcription factor family protein \\
\hline HORVU5Hr1G045640 & $\mathrm{n} / \mathrm{c}$ & $\mathrm{n} / \mathrm{c}$ & down & down & NAC & NAC domain protein, \\
\hline HORVU2Hr1G072420 & $\mathrm{n} / \mathrm{c}$ & $\mathrm{n} / \mathrm{c}$ & down & $\mathrm{n} / \mathrm{c}$ & S1Fa-like & DNA-binding protein S1FA2 \\
\hline HORVU3Hr1G094730 & $\mathrm{n} / \mathrm{c}$ & $\mathrm{n} / \mathrm{c}$ & down & $\mathrm{n} / \mathrm{c}$ & SBP & Squamosa promoter-binding-like protein 2 \\
\hline HORVU1Hr1G054620 & $\mathrm{n} / \mathrm{c}$ & $\mathrm{n} / \mathrm{c}$ & down & $\mathrm{n} / \mathrm{c}$ & STAT & SH2 domain protein B \\
\hline HORVU3Hr1G095400 & $\mathrm{n} / \mathrm{c}$ & $\mathrm{n} / \mathrm{c}$ & down & down & TCP & TCP family transcription factor \\
\hline HORVU6Hr1G093960 & $\mathrm{n} / \mathrm{c}$ & $\mathrm{n} / \mathrm{c}$ & down & $\mathrm{n} / \mathrm{c}$ & TCP & TCP family transcription factor \\
\hline
\end{tabular}


Table 3. Cont

\begin{tabular}{|c|c|c|c|c|c|c|}
\hline \multirow[b]{2}{*}{ Horvu ID } & \multicolumn{4}{|c|}{ Expression change } & \multirow[b]{2}{*}{ Family Name } & \multirow[b]{2}{*}{ Gene Description } \\
\hline & CamB Roots & CamB Leaves & Mar Roots & Mar Leaves & & \\
\hline HORVU7Hr1G038130 & $\mathrm{n} / \mathrm{c}$ & $\mathrm{n} / \mathrm{c}$ & down & $\mathrm{n} / \mathrm{c}$ & TCP & TCP family transcription factor \\
\hline HORVU7Hr1G041230 & $\mathrm{n} / \mathrm{c}$ & $\mathrm{n} / \mathrm{c}$ & down & up & Tify & Protein TIFY $3 \mathrm{~A}$ \\
\hline HORVU1Hr1G069620 & $\mathrm{n} / \mathrm{c}$ & $\mathrm{n} / \mathrm{c}$ & down & $\mathrm{n} / \mathrm{c}$ & WRKY & WRKY DNA-binding protein 54 \\
\hline HORVU3Hr1G050590 & $\mathrm{n} / \mathrm{c}$ & $\mathrm{n} / \mathrm{c}$ & down & $\mathrm{n} / \mathrm{c}$ & WRKY & WRKY DNA-binding protein 50 \\
\hline \multicolumn{7}{|c|}{ Transcriptional regulators: } \\
\hline HORVU6Hr1G091700 & $\mathrm{n} / \mathrm{c}$ & $\mathrm{n} / \mathrm{c}$ & down & $\mathrm{n} / \mathrm{c}$ & Others & Ethylene receptor 3 \\
\hline HORVU2Hr1G017680 & $\mathrm{n} / \mathrm{c}$ & up & down & up & PHD & E3 ubiquitin-protein ligase SHPRH \\
\hline HORVU4Hr1G003040 & $\mathrm{n} / \mathrm{c}$ & $\mathrm{n} / \mathrm{c}$ & down & $\mathrm{n} / \mathrm{c}$ & TRAF & Regulatory protein (NPR1) \\
\hline
\end{tabular}




\subsection{Target Genes Putatively Regulated by Specific Transcription Factors with Differential Expression in Roots}

To depict some possible regulatory connections between specific transcription factors and their putative target genes, we have subjected all genes differentially expressed in roots of both genotypes to the analysis in the Transcription Factor Enrichment tool (http://plantregmap.cbi.pku.edu.cn) that allows us to find the TFs which possess significantly over-represented targets in the input gene list. For this purpose, the whole list of root DEGs was divided according to the direction of their expression change, and then the two sets of genes were used as queries against the TF enrichment tool. It was possible to find four TF genes that were differentially expressed after the mild drought stress in roots and possessed overrepresented targets in our data set (Table 4 and Table S8). Three of them were up-regulated, and one (MLOC_81003, encoding a TF from bZIP family) was down-regulated by the stress in both genotypes. All their putative target genes had the same direction of expression change-up-regulation, which indicates that the MLOC_81003 gene may act as a negative regulator of these putative targets.

Table 4. The transcription factors that possess significantly over-represented targets within root DEGs.

\begin{tabular}{|c|c|c|c|c|c|c|c|c|}
\hline Horvu ID & MLOC ID & $\begin{array}{l}\text { Background- } \\
\text { All }^{1}\end{array}$ & $\begin{array}{l}\text { Background- } \\
\text { Bind }^{2}\end{array}$ & Query-All $^{3}$ & $\begin{array}{l}\text { Query- } \\
\text { Bind }^{4}\end{array}$ & $p$-Value & $q$-Value & $\begin{array}{l}\text { Description } \\
\text { (IPK) }\end{array}$ \\
\hline HORVU1Hr1G090030 & MLOC_6711 & 24306 & 470 & 546 & 21 & $1.059 \times 10^{-3}$ & $3.352 \times 10^{-2}$ & $\begin{array}{c}\text { G-box } \\
\text { binding } \\
\text { factor } 2\end{array}$ \\
\hline HORVU5Hr1G014170 & MLOC_81003 & 24306 & 388 & 546 & 16 & $7.159 \times 10^{-3}$ & $9.715 \times 10^{-2}$ & $\begin{array}{c}\text { Basic-leucine } \\
\text { zipper (bZIP) } \\
\text { TF family } \\
\text { protein }\end{array}$ \\
\hline HORVU6Hr1G068100 & MLOC_14844 & 24306 & 386 & 546 & 14 & $2.906 \times 10^{-2}$ & $2.301 \times 10^{-1}$ & $\begin{array}{l}\text { Myb-like TF } \\
\text { family } \\
\text { protein }\end{array}$ \\
\hline
\end{tabular}

We also found that some regulatory cascades may exist between the identified TF genes. The MLOC_14844 encoding a TF from Myb-like family was within the targets of MLOC_6711, a gene for a G-box binding factor 2, belonging to the bZIP family, indicating a possible regulatory connection between these two genes. The latter may be under a negative regulation of MLOC_81003 (another bZIP TF), and a MLOC_75166 encoding heat stress TF may be auto-regulated as this gene was found among its own targets (Figure 3, Table S8). This regulatory network may be further extended based on other identified target genes. The analysis showed that MLOC_14844 may regulate the expression of another G-box binding transcription factor (MLOC_15316). The MLOC_6711 may be responsible for the activation of a gene from C3H family (MLOC_63525), and MLOC_75166 may regulate another two TF-encoding genes representing NAC family (MLOC_37104 and MLOC_23616), The last one was annotated as a protein with unknown function, but it possesses domains characteristic to NACs (Table S8).

The function of the putative targets for the four TFs spans several categories, such as metabolism, including nucleic acid metabolism, gene expression regulation with the above-mentioned transcription factors, and other molecules involved in transcription regulation, translation initiation, or ribosomal proteins. Other genes belong to stress response signaling and redox processes, transmembrane transport or plastid formation. We have not found any specific preferences of the four TFs to target genes from a defined biological process; they may all regulate expression of genes of the broad spectrum of biological functions. 


\begin{tabular}{|c|c|c|c|c|}
\hline TF & \multicolumn{4}{|c|}{ 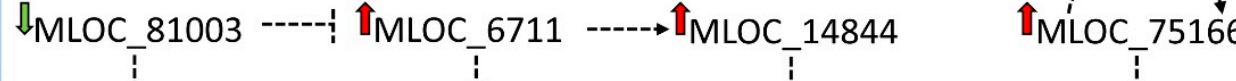 } \\
\hline \multirow{18}{*}{ 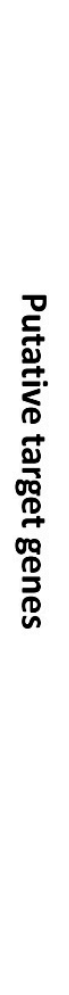 } & MLOC_21885 & MLOC_14214 & MLOC_32633 & MLOC_20639 \\
\hline & MLOC_3114 & MLOC_14884* & MLOC_4480 & MLOC_23616 \\
\hline & MLOC_37693 & MLOC_15021 & MLOC_66700 & MLOC_37104* \\
\hline & MLOC_47741 & MLOC 21885 & MLOC_80071 & MLOC_44536 \\
\hline & MLOC_51183 & MLOC_26641 & MLOC_28425 & MLOC_6125 \\
\hline & MLOC_55348 & MLOC_3982 & MLOC_52874 & MLOC_74277 \\
\hline & MLOC_60073 & MLOC_55553 & MLOC_66714 & MLOC_75166* \\
\hline & MLOC_60376 & MLOC_60073 & MLOC_16587 & MLOC_78762 \\
\hline & MLOC_6125 & $\begin{array}{l}\text { MLOC_6125 } \\
\text { MIOC } 61260\end{array}$ & MLOC_29951 & MLOC_79789 \\
\hline & MLOC_61260 & MLOC $63525^{*}$ & MLOC_55858 & MLOC_60937 \\
\hline & MLOC_6711* & MLOC_77985 & MLOC_57145 & MLOC_24040 \\
\hline & MLOC_68719 & MLOC_8076 & MLOC_62551 & MLOC_72096 \\
\hline & MLOC_77985 & MLOC_60937 & MLOC_67686 & MLOC_77485 \\
\hline & MLOC 8076 & MLOC_61601 & & MLOC 82019 \\
\hline & MIOC 61601 & MLOC_64408 & & \\
\hline & & MLOC_22824 & & \\
\hline & & MLOC_3858 & & \\
\hline & & MLOC 78806 & & \\
\hline
\end{tabular}

Figure 3. Putative targets and predicted regulatory network of these TFs, which possess significantly over-represented targets within root DEGs identified after mild drought stress. Violet-DEGs common to CamB and Mar genotypes; orange-DEGs specific to Maresi; blue-DEGs specific to CamB. Asterisk-DEGs encoding TFs.

2.5. A Comparison of DEGs Involved in Gene Expression Regulation in Roots during Mild and Severe Drought Stress

The drought response on the transcriptional level of the same barley genotypes (CamB and Maresi) was previously analyzed under severe drought stress, where the seedlings at the same stage as presented here were grown for 10 days in the soil moisture close to the point of permanent plant wilting [20]. Thus, it was possible to check which genes involved in gene expression regulation in roots are common or specific to the different drought regimes.

Altogether, the severe drought stress resulted in the expression changes of 187 DEGs encoding transcription factors that belong to 47 different families and 43 DEGs encoding other proteins involved in transcription regulation from 12 different families. Of this number, 42 DEGs were common for severe and mild drought stress, including 38 DEGs for transcription factors and 4 DEGs for other regulators of gene expression. Mild drought-specific DEGs included 47 genes, and 40 of them encode transcription factors. The remaining 188 DEGs were specific to the severe drought stress (149 genes for TFs and 39 genes for other types of expression regulators; Figure 4, Table S9). 


\begin{tabular}{|c|c|}
\hline $\begin{array}{l}\text { Family } \\
\text { name }\end{array}$ & $\begin{array}{c}\text { Mild vs severe } \\
\text { drought DEGs } \\
\text { distribution }\end{array}$ \\
\hline $\begin{array}{c}\text { AP2/ERF- } \\
\text { ERF }\end{array}$ & \\
\hline $\begin{array}{c}\text { AP2/ERF- } \\
\text { RAV }\end{array}$ & \\
\hline$A \cup X / I A A$ & 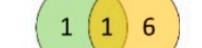 \\
\hline B3 & 03 \\
\hline B3-ARF & 3 \\
\hline $\begin{array}{c}\text { BAH } \\
\text { domain }\end{array}$ & \\
\hline BES1 & 2 \\
\hline bHLH & 6 \\
\hline bZIP & 12 \\
\hline $\begin{array}{l}\mathrm{C} 2 \mathrm{C} 2-\mathrm{CO}- \\
\text { like }\end{array}$ & \\
\hline C2C2-Dof & 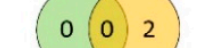 \\
\hline $\begin{array}{l}\text { C2C2- } \\
\text { GATA }\end{array}$ & \\
\hline C2C2-LSD & 1 \\
\hline $\mathrm{C} 2 \mathrm{H} 2$ & 8 \\
\hline $\mathrm{C} 3 \mathrm{H}$ & 1 \\
\hline CPP & 10 \\
\hline CSD & 00 \\
\hline
\end{tabular}

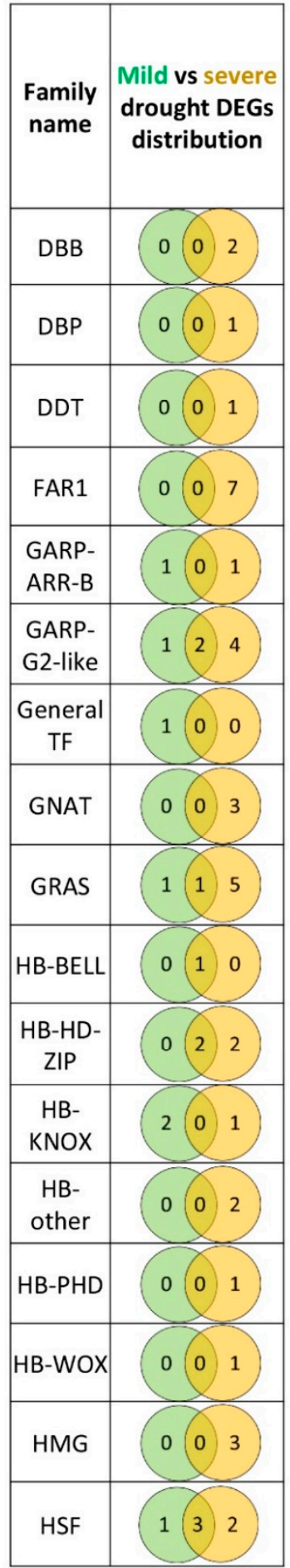

\begin{tabular}{|c|c|}
\hline $\begin{array}{c}\text { Family } \\
\text { name }\end{array}$ & $\begin{array}{l}\text { Mild vs severe } \\
\text { drought DEGs } \\
\text { distribution }\end{array}$ \\
\hline IWS1 & (0) 1 \\
\hline LIM & 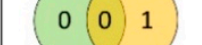 \\
\hline LOB & 3 \\
\hline $\begin{array}{l}\text { MADS- } \\
\text { M-type }\end{array}$ & \\
\hline $\begin{array}{l}\text { MADS- } \\
\text { MIKC }\end{array}$ & \\
\hline mTERF & 3 \\
\hline MYB & 8 \\
\hline $\begin{array}{c}\text { MYB- } \\
\text { related }\end{array}$ & \\
\hline NAC & 12 \\
\hline$N F-Y A$ & 3 \\
\hline NF-YB & 1 \\
\hline NF-YC & 1 \\
\hline OFP & 1 \\
\hline Others & 01 \\
\hline PHD & 2 \\
\hline PLATZ & 0 \\
\hline $\begin{array}{c}\text { Pseudo } \\
\text { ARR-B }\end{array}$ & 0 \\
\hline
\end{tabular}

\begin{tabular}{|c|c|}
\hline $\begin{array}{l}\text { Family } \\
\text { name }\end{array}$ & $\begin{array}{l}\text { Mild vs severe } \\
\text { drought DEGs } \\
\text { distribution }\end{array}$ \\
\hline RWP-RK & 0 \\
\hline $\begin{array}{l}\text { S1Fa- } \\
\text { like }\end{array}$ & \\
\hline SBP & \\
\hline $\begin{array}{l}\text { SCAI TF } \\
\text { family }\end{array}$ & \\
\hline SET & 0 \\
\hline SNF2 & 00 \\
\hline $\begin{array}{l}\text { SWI/SNF } \\
\text {-BAF60b }\end{array}$ & \\
\hline STAT & \\
\hline TCP & \\
\hline Tify & \\
\hline TRAF & \\
\hline Trihelix & 8 \\
\hline TUB & 01 \\
\hline VOZ & 00 \\
\hline WRKY & 26 \\
\hline
\end{tabular}

Figure 4. An overview of the number of TFs genes differentially expressed in roots after mild and severe drought treatment.

Factors from AP2/ERF family, B3, and bHLH families predominated within the group of DEGs specific to mild drought (Figure 4). Moreover, genes from MADS-M-type, S1Fa-like, SBP, SCAI, and STAT families were differentially expressed after the mild drought stress only. On the other hand, the severe drought stress resulted in a specific differential expression of genes from 20 TF families and the most numerous were the $\mathrm{C} 2 \mathrm{H} 2$, Far1, and Trihelix families. Many DEGs specific to severe drought also belong to the AP2/ERF, bZIP, bHLH, NAC, and WRKY families. 
Within DEGs common to both stresses, again, factors from AP2/ERF, bZIP, bHLH families were found, together with genes belonging to the heat shock, NAC and WRKY families, among others. Importantly, the pattern of gene expression changes after mild and severe stress or between the two genotypes was not uniform for all DEGs (Figure 5, Table S10) and interestingly, we found some DEGs that showed opposite expression change in both drought regimes: up-regulation after mild and down-regulation after severe drought (Table 5). All of these DEGs, but one, were specific to roots, they did not change their expression in leaves in either of drought regimes.

\begin{tabular}{|c|c|c|c|c|c|}
\hline \multirow[b]{2}{*}{ Gene_ID (HORVU) } & \multirow[b]{2}{*}{ Family Name } & \multicolumn{2}{|c|}{ Cam Root } & \multicolumn{2}{|c|}{ Mar Root } \\
\hline & & $\begin{array}{c}\text { Mild } \\
\text { drought }\end{array}$ & $\begin{array}{c}\text { Severe } \\
\text { drought }\end{array}$ & $\begin{array}{c}\text { Mild } \\
\text { drought }\end{array}$ & $\begin{array}{c}\text { Severe } \\
\text { drought }\end{array}$ \\
\hline HORVU3Hr1G010190 & AP2/ERF-ERF & & & & \\
\hline HORVU1Hr1G091540 & MYB-related & & & & \\
\hline HORVU5Hr1G065420 & WRKY & & & & \\
\hline HORVU1Hr1G090250 & AP2/ERF-ERF & & & & \\
\hline HORVUOHr1G013950 & bZIP & & & & \\
\hline HORVU4Hr1G021720 & bZIP & & & & \\
\hline HORVU3Hr1G019510 & $\mathrm{C} 3 \mathrm{H}$ & & & & \\
\hline HORVU2Hr1G014590 & HSF & & & & \\
\hline HORVU7Hr1G056820 & HSF & & & & \\
\hline HORVU2Hr1G082330 & NAC & & & & \\
\hline HORVU5Hr1G111590 & NAC & & & & \\
\hline HORVU7Hr1G089930 & AP2/ERF-ERF & & & & \\
\hline HORVU2Hr1G017460 & NAC & & & & \\
\hline HORVU5Hr1G034830 & WRKY & & & & \\
\hline HORVU7Hr1G075960 & WRKY & & & & \\
\hline HORVU4Hr1G080460 & GRAS & & & & \\
\hline HORVU1Hr1G081570 & WRKY & & & & \\
\hline HORVU6Hr1G071950 & C2C2-CO-like & & & & \\
\hline HORVU3Hr1G052710 & MYB-related & & & & \\
\hline HORVU5Hr1G045640 & NAC & & & & \\
\hline HORVU3Hr1G108680 & bHLH & & & & \\
\hline HORVU2Hr1G020140 & GARP-G2-like & & & & \\
\hline HORVU3Hr1G085720 & HB-BELL & & & & \\
\hline HORVU5Hr1G067010 & HB-HD-ZIP & & & & \\
\hline HORVUOHr1G003020 & MADS-MIKC & & & & \\
\hline HORVU7Hr1G038130 & TCP & & & & \\
\hline HORVU1Hr1G069620 & WRKY & & & & \\
\hline HORVU3Hr1G061930 & TUB & & & & \\
\hline HORVU1Hr1G071620 & NF-YB & & & & \\
\hline HORVU7Hr1G055180 & bHLH & & & & \\
\hline HORVU1Hr1G051260 & WRKY & & & & \\
\hline HORVU7Hr1G026940 & AP2/ERF-ERF & & & & \\
\hline HORVU1Hr1G086580 & bZIP & & & & \\
\hline HORVU1Hr1G055930 & HB-HD-ZIP & & & & \\
\hline HORVU7Hr1G088920 & HSF & & & & \\
\hline HORVU2Hr1G041300 & MYB & & & & \\
\hline HORVU5Hr1G047610 & LOB & & & & \\
\hline HORVU7Hr1G096430 & GARP-G2-like & & & & \\
\hline
\end{tabular}

Figure 5. The expression pattern of DEGs encoding transcription factors, which were differentially expressed in roots of barley seedlings subjected to mild and severe drought regimes. Green: down-regulation of a gene; red: up-regulation of a gene. 
Table 5. Genes that show an opposite expression pattern under mild and severe drought stress.

\begin{tabular}{|c|c|c|c|c|c|c|c|c|c|c|c|}
\hline \multirow{3}{*}{ Horvu ID } & \multirow{3}{*}{ MLOC ID } & \multirow{3}{*}{ Family Name } & \multicolumn{4}{|c|}{ Mild Drought } & \multicolumn{4}{|c|}{ Severe Drought } & \multirow{3}{*}{ Description } \\
\hline & & & \multicolumn{2}{|c|}{ Roots } & \multicolumn{2}{|c|}{ Leaves } & \multicolumn{2}{|c|}{ Roots } & \multicolumn{2}{|c|}{ Leaves } & \\
\hline & & & CamB & Mar & CamB & Mar & CamB & Mar & CamB & Mar & \\
\hline HORVU7Hr1G089930 & MLOC_59305 & AP2/ERF-ERF & up & up & $\mathrm{n} / \mathrm{c}$ & $\mathrm{n} / \mathrm{c}$ & down & down & $\mathrm{n} / \mathrm{c}$ & $\mathrm{n} / \mathrm{c}$ & Ethylene-responsive transcription factor 1 \\
\hline HORVU5Hr1G047610 & MLOC_5148 & LOB & up & up & $\mathrm{n} / \mathrm{c}$ & $\mathrm{n} / \mathrm{c}$ & $\mathrm{n} / \mathrm{c}$ & down & $\mathrm{n} / \mathrm{c}$ & $\mathrm{n} / \mathrm{c}$ & LOB domain-containing protein 4 \\
\hline HORVU2Hr1G017460 & MLOC_65101 & NAC & up & up & $\mathrm{n} / \mathrm{c}$ & up & down & down & & up & NAC domain protein \\
\hline HORVU1Hr1G081570 & MLOC_32433 & WRKY & up & up & $\mathrm{n} / \mathrm{c}$ & $\mathrm{n} / \mathrm{c}$ & down & $\mathrm{n} / \mathrm{c}$ & $\mathrm{n} / \mathrm{c}$ & $\mathrm{n} / \mathrm{c}$ & WRKY DNA-binding protein 24 \\
\hline HORVU3Hr1G108680 & MLOC_36351 & bHLH & $\mathrm{n} / \mathrm{c}$ & up & $\mathrm{n} / \mathrm{c}$ & $\mathrm{n} / \mathrm{c}$ & down & down & $\mathrm{n} / \mathrm{c}$ & $\mathrm{n} / \mathrm{c}$ & Transcription factor ORG2 \\
\hline
\end{tabular}




\section{Discussion}

Our analysis of root transcriptomes of two barley genotypes, which differed in their level of drought tolerance, allowed us to select several genes encoding transcription factors that may be involved in better drought survival. Among the ten genes that were specifically activated or repressed in roots of drought-tolerant $\mathrm{CamB}$, one encodes the TSO1-like CXC protein, which is the homolog of the human TESMIN gene that is expressed mainly in testes and ovaries [21]. In Arabidopsis, the TSO1 gene functions in floral tissue development, inflorescence meristem organization, and control of cell division [22]. Importantly, it is required for the correct organization of shoot and root apical meristems [23]. It was shown that TSO1 acts as a repressor of the MYB3R1 gene, which in turn maintains the repressed state of G2/M-specific genes in the roots, preventing cell proliferation. Thus, high expression of TSO1 allows the maintenance of root proliferation in the root meristematic zone [23]. In our study, the up-regulation of the TSO1-like gene in CamB roots suggests that it may be involved in the promotion of better drought tolerance of this genotype.

In our data set, no differential expression of the MYB3R1 homologue was noticed, but we found two other genes from the MYB superfamily, belonging to the GARP-G2-like group that were specific to CamB roots (HORVU2Hr1G020140 and HORVU7Hr1G096430). The first one was up-regulated and was annotated as two-component response regulator ARR18, but the homology search in Ensembl Plants database showed that its orthologues in Arabidopsis (HRS1, HHO2, or HHO3) and rice (NIGT1) are involved in nitrate and phosphate signaling in roots [24] and in the presence of nitrate they repress the nitrate-starvation response [25]. The second one was down-regulated in CamB roots and is the orthologue of Arabidopsis PHL6 and rice PHR4 genes, that are activated upon limiting phosphate availability and regulate phosphate-starvation response pathway [26]. It was shown that high nitrate reduces lateral root elongation [27] and a similar observation was true for drought treated plants [28]. On the other hand, phosphorus starvation increases lateral root formation and the density of root hairs [27], placing these genes in a pathway that may influence the root plasticity in response to the soil environment. Expression pattern of barley orthologues of NIGH1 and PHR4 observed in our study suggests that they may also play a role in drought response although their precise function in this type of stress remains to be elucidated.

An interesting gene (HORVU5Hr1G062040) that was up-regulated in CamB roots shows similarity to human SCAI, the suppressor of cancer cell invasion gene that inhibits the invasive migration of tumor cells [29]. SCAI protein interacts with KDM3B, a histone demethylase protein that removes methyl groups from $\mathrm{H} 3 \mathrm{~K} 9 \mathrm{me} 1 / 2$, which is the mark of repressed chromatin state [30], and with the SWI/SNF chromatin remodeling complex [31]. Thus, the HORVU5Hr1G062040 gene plays a role in the regulation of chromatin status and its transcriptional availability. Two other DEGs involved in epigenetic regulation were found in CamB roots: a down-regulated HORVU5Hr1G061120 gene encoding a bromo-adjacent homology $(\mathrm{BAH})$ domain-containing protein and an up-regulated HORVU6Hr1G034680 gene for a methyl-CpG-binding domain-containing protein. The proteins with a BAH domain recognize methylated state of histone $\mathrm{H} 3$ lysines that are the hallmarks of the heterochromatin [32]. The methyl-CpG-binding proteins interact with methylated CpG sites in DNA and are also responsible for silencing the target chromatin regions [33]. The direction of expression changes of these genes in the roots of the CamB genotype suggests that the efficient response to drought may require the formation of an active chromatin status of genes that are targeted by SCAI and BAH proteins. The repression of transcription of other genes, which are under the regulation mediated by the methyl-CpG-binding protein, may be also needed.

In the roots of Maresi, contrary to CamB, more genotype-specific DEGs for gene expression regulatory proteins were found, and the great majority of them were down-regulated by the stress. This finding suggests that in a drought-susceptible cultivar, more dynamic changes occur after the drought treatment. It is not clear whether the prevalence of down-regulatory mechanisms is an indicator of a lower acclimation ability to the stress or it just suggests that Maresi enters a substantially different pathway of drought response than CamB. Many detected TFs belong to large families with 
members of positive and negative expression regulatory functions, and there are reports of their both up- or down-regulation upon drought. Such observations were made, for example, for genes from AP2/ERF [34,35], bHLH, MADS, MYB [8], NAC [9], or WRKY families [7]. Thus, a more detailed analysis targeted to single genes is needed to detect their specific impact on drought response in the Maresi cultivar. Nevertheless, some possible molecular response pathways have emerged that may be characteristic of the roots of this drought-susceptible genotype.

One pathway may be connected to jasmonic acid (JA) signaling and the action of three genes: HORVU7Hr1G041230, HORVU3Hr1G050590, and HORVU4Hr1G003040 encoding a TIFY3A TF, WRKY and an NPR1 protein, respectively. The TIFY3A TF belongs to the JAZ subfamily of TIFY, which is known to repress the action of Arabidopsis MYC2 TF and its homologues-the positive regulators of JA-dependent responses. The important effect of such response is an inhibition of primary root growth [36]. The involvement of TIFY TFs in drought tolerance was demonstrated by the overexpression study of rice OsTIFY11a, which resulted in the enhancement of dehydration stress resistance [37]. Down-regulation of this gene in Maresi roots suggests that this pathway of possible drought tolerance may be negatively affected in this cultivar.

Similar, negative regulation of JA response was found in Arabidopsis for another factor, WRKY50 [38], a homologue of which HORVU3Hr1G050590 was again down-regulated in roots of Maresi. Another down-regulated gene (HORVU4Hr1G003040), encoding a regulatory protein NPR1, may also be placed within the JA-related response. It is a repressor of JA signaling that acts through the induction of the salicylic acid pathway [39], and its overexpression in Arabidopsis leads to oxidative stress resistance [40]. Taking all of this together, down-regulation of the above-mentioned genes may lead to the lack or lower rate of JA signaling repression in Maresi roots, which in turn may diminish the efficient drought response.

Nonetheless, more complexity to the image of this response is added by the down-regulation of two KNOTTED1-like (KN1) transcription factor genes (HORVU2Hr1G061320 and HORVU5Hr1G098570). The KN1 TF negatively modulates the accumulation of gibberellin (GA) through the control of ga2ox1, which is an enzyme that leads to the catalysis of the GA [41]. Thus, down-regulation of KN1 genes in Maresi roots may slow-down the degradation of GA, which in turn may promote root cell proliferation and elongation [42]. Moreover, it was shown that there is a crosstalk between GA and JA, mediated by MYC and DELLA proteins that compete with each other to bind with JAZ TF. In the presence of GA, DELLA protein is directed to degradation, and then JAZ and MYC may interact with each other, which prevents MYC from activation of JA-dependent genes and leads to the repression of JA-driven response [43]. This complex response of Maresi indicates the necessity of a substantial rebuilding of root metabolism under the mild drought in a susceptible cultivar, which does not take place in a tolerant genotype.

Based on the enrichment analysis, we performed a prediction of possible relationships between differentially expressed TF genes and all DEGs that were identified in the roots of both genotypes. We found four TFs that had overrepresented targets among root DEGs, which may place them near the top of the regulatory network of the mild drought response in roots. Two of them encode TFs from the bZIP family: MLOC_81003 (HORVU5Hr1G014170) with the highest similarity to rice bZIP88 and MLOC_6711 (HORVU1Hr1G090030), similar to Arabidopsis G-box binding factor 2 (GBF2). Many bZIP TFs are involved in stress signaling and act in an ABA-dependent manner [44]. Our data suggest a possible negative regulation of GBF2 by bZIP88 (HORVU5Hr1G014170) in drought response in barley roots, as the up-regulated GBF2 gene was found among targets of down-regulated bZIP88. A down-regulation of $b$ ZIP88 was also observed in rice subjected to various abiotic stresses, including drought [45]. Interestingly, a DNA binding activity of some G-group bZIPs was found to be regulated by light and reactive oxygen species (ROS) [46], which are commonly produced in tissues upon the abiotic stresses. Thus, one may hypothesize that ROS produced in drought treated roots may trigger the regulation of gene expression downstream of GBF2. Another TF gene with overrepresented targets encodes heat stress factor (HORVU7Hr1G056820) orthologous to rice, HSFB-2b gene. HSFs from 
class B are considered to be transcriptional repressors [47], contrary to our enrichments analysis data, which pointed to several up-regulated DEGs downstream to the HFSB2b gene (also up-regulated). On the other hand, a study of seed germination under heat stress in Arabidopsis showed the induction of the HSFB2b gene, which was accompanied by the accumulation of 49 heat shock proteins [48] (HSP). In our analysis, two up-regulated genes (HORVU3Hr1G007500 and HORVU3Hr1G020520) encoding HSPs were found as targets of HSFB2b. Moreover, a study of maize transcriptome suggests that the HSFB2b gene may be important in drought tolerance in this species, as HSFB2b expression was specifically activated in a drought-tolerant genotype [49]. Nevertheless, at this stage of our analysis, we treat the above enrichment data only as putative interactions that may serve to drive hypothesis on novel regulatory connections in the gene network, and which need to be verified based on gene-specific studies.

The comparison of mild and severe drought stress regimes showed a significant increase in the number of DEGs involved in gene expression regulation when a severe drought was applied. Among all the regulatory DEGs detected after mild drought treatment, about 50\% were also differentially expressed after severe stress. Additionally, the number of DEGs that were specific to only one stress regime was four times higher after severe stress, compared to the mild drought. Such results suggest that a substantial part of root drought response that is initiated upon moderate stress may also persist in more severe conditions. It is important to emphasize, however, that the details of this response differed in a genotype-dependent manner. A general scheme that emerged from our analysis suggests that the drought-sensitive genotype goes through more profound changes in the transcriptome than the drought-tolerant form, especially after the severe stress. The differences between the two drought regimes that were observed for DEGs with expression regulatory function were also reflected in the expression of all remaining genes involved in the other biological processes. Gene ontology analysis showed that DEGs identified after mild drought belong to similar biological and functional categories as genes differentially expressed after severe stress [20]. However, when we take into consideration the number of DEGs, then more DEGs are found within each GO category when the stress is stronger, and again, more DEGs are present in the drought-sensitive genotype [20]. The same was true for the TF DEGs detected in the presented study-a much higher number of TF DEGs were found after severe stress in each TF family, and this increase in number was more prominent in the Maresi genotype. A remarkably higher number of DEGs characteristic for severe drought were noticed, for example, within the AP/ERF family of ethylene responsive factors and AUX/IAA family involved in auxin signaling. A similar increase in gene number was noticed for the bZIP family, including factors involved in ABA signaling. It was shown that both hormones enter a crosstalk important for a drought response and trigger the inhibition of lateral root formation [50], making their regulatory network essential for shaping the root system architecture under stress. Severe stress also resulted in a higher number of genes from GRAS family, which may be placed within gibberellin signaling [51] and the genes from the Tify family involved in jasmonate signaling [52]. Interestingly, the Tify TFs detected under severe stress included down-regulated genes in $\mathrm{CamB}$, suggesting that the drought-tolerant genotype may enter a similar response pathway related to jasmonates as the sensitive cultivar, but only after much more adverse environmental conditions.

An interesting finding was the detection of five genes, which exhibited opposite expression changes after mild and severe stresses, and in the case of two of them, some functional characteristics may be related to the root function under drought. The first one (HORVU7Hr1G089930), which encodes a TF from the AP/ERF family is an orthologue of the Arabidopsis gene for the SHINE factor that is involved in wax synthesis. Overexpression of this gene caused the increase of wax biosynthesis and altered its composition, resulting in a higher cuticle permeability in leaves and better drought tolerance [53]. The Arabiopsis SHINE gene is also expressed in roots and is probably involved in suberin deposition [54]. Suberisation is considered to have a negative impact on water and solute transport, but it may also prevent an uncontrolled backflow of water to the surrounding soil under unfavorable environmental conditions [55]. During the day and in optimal soil moisture, the main 
force driving water transport is the transpiration, and water is transported via the apoplast. During the night or when stomata are closed due to drought stress, water flow from cell-to-cell and is driven by the root pressure. In the latter scenario, the suberin acts as a barrier limiting apoplastic transport [56]. The barley SHINE orthologue was up-regulated under mild drought in our study, but down-regulated after the severe water shortage conditions in both genotypes. It is possible that SHINE allows the increase of suberization when the signal of water shortage is perceived by the roots, and consequently, it helps to actively maintain the transport of limited, but still available water to the shoot, even if the transpiration rate is reduced. On the other hand, under the severe drought, when water is very limited, a high deposition of suberin may be unfavorable as it may increase hydraulic resistance of the root to the extent it prevents water acquisition.

The second gene with the opposite expression change under moderate and severe drought (HORVU5Hr1G047610) belongs to the LOB (LATERAL ORGAN BOUNDARIES) family and was also up-regulated after mild drought in both genotypes and down-regulated in severe drought, but only in the Maresi cultivar. LOB transcription factors are involved in the development of lateral organs. The first LOB gene identified in Arabidopsis is expressed at the boundaries between stem cells and developing leaf primordia at shoot apical meristem [57]. Several $L O B$ genes are important for lateral root formation driven by auxin signaling and modulated by ABA under stress conditions [58,59]. A maize RTCS gene from this family is responsible for the initiation and maintenance of embryonic seminal roots and shoot-born root primordia [60], and the ARL1 gene in rice is involved in adventitious roots formation [61]. At present, it is difficult to predict the precise function of the $L O B$ gene identified in the presented study in barley, but its differential expression in roots only and not in leaves suggests its importance for shaping root architecture and building an appropriate root system under the moderate but not in the severe stress conditions.

Another aspect that emerged from our transcriptomic study was the discovery of genes that function in the photosynthesis process, but were differentially expressed under drought in roots of one or both barley genotypes. They included genes encoding proteins of oxygen-evolving complex, together with proteins that build photosystem I (PSI) and PSII in leaf chloroplasts, and most of them were up-regulated by drought stress. This finding is in agreement with our previous study, where several genes annotated as photosynthesis-related were also differentially expressed in roots upon severe drought [20]. We hypothesize that such result may suggest a specific role of root plastids as ROS scavenging centers, which may help root cells to cope with oxidative stress conditions generated by drought. Differential expression of genes involved in photosynthesis in roots under drought is not uncommon and was also detected in other studies, for example, in cotton [62], chickpea [63] poplar [64], and pine [65].

Our analysis also suggests that under the mild drought stress, different genes involved in photosynthesis are differentially expressed in leaves and the roots. We found, for example, up-regulation of a gene for RuBisCO activase A in Maresi leaves and differential expression of two genes encoding chlorophyll $\mathrm{a}-\mathrm{b}$ binding proteins in leaves of both genotypes, whereas another gene for chlorophyll $\mathrm{a}-\mathrm{b}$ binding protein was up-regulated in roots only. Similarly, we noticed down-regulation of a gene encoding one of PSII components in leaves, whereas, in roots, two other genes for PSII proteins were up-regulated. This may suggest that drought results in an organ-specific expression of genes with similar function, although we are aware that we analyzed only one time-point of the stress treatment, so we only spotted a subset of the dynamic changes of the transcriptomes and we cannot rule out that some of these genes exhibit different expression patterns at the early stage of the stress. Such possibility is partially supported by the research of Chmielewska et al. [18] who used plant material from the same experiment (the same genotypes and drought treatment) for the analysis of leaves and roots proteomes and metabolomes. On the protein level, they found a higher accumulation of oxygen-evolving enhancer protein in leaves of Maresi and a ferredoxin-NADP+ oxidoreductase in leaves of CamB, while in our study the differential expression of their transcripts was not found. It is likely that the up-regulation of transcription could occur at an earlier stage of the stress application 
and on the 10th day, the differences were visible only on the protein level. Similarly, Chmielewska et al. [18] found a higher accumulation of RuBisCO activase B in both genotypes and RuBisCO activase A protein in the leaves of Maresi only, while in our analysis, only the latter protein is in agreement with its transcript up-regulation. Nevertheless, taking together the above aspects of our analysis, the photosynthesis-related genes may be considered as a source of better drought response of roots, due to their possible anti-oxidative role protecting macromolecules from oxidative damage in this organ.

To conclude, we have found a substantial number of transcription factor encoding genes that are involved in the drought response in roots of barley. The use of microarrays, despite their limitations, allowed us to make an unbiased comparison of transcriptome changes induced by mild drought to the response of the same genotypes to severe stress, which we studied previously [20]. The comparability of both analyses was ensured by the use of the same type of arrays and experimental methodology, except for the drought regime, in both experiments. We are aware, however, that additional use of the RNA-Seq method may give a more in-depth view on global gene expression changes. Nevertheless, our analysis suggests that genotype-dependent drought tolerance is accompanied by a lower number of gene expression changes than response of the susceptible genotype. The gene expression regulation is probably directed toward the maintenance of root meristems proliferation and a yet unidentified network of epigenetic changes that may allow a better acclimation of the plant to the stress conditions and may be triggered by factors involved in chromatin remodeling and DNA methylation. We found that a part of the drought response mechanisms is controlled by transcription regulators from Tiffy, WRKY, HB-KNOX, and mTERF families that may act within jasmonate and gibberellin signaling pathways. Our data also show that the activation of several TFs may be important in mild drought but not in severe drought response. We believe that the TF-encoding genes identified in the present study may be used as future targets for the enhancement of drought tolerance in barley and related species.

\section{Materials and Methods}

\subsection{Plant Material and STRESS Treatment}

Two barley genotypes were used for the study: a European semidwarf cultivar Maresi and a Syrian breeding line Cam/B1/CI08887//CI05761 (CamB), adapted to dry environments. Grains of both genotypes were sterilized, and initially, 20 grains were placed in the double-walled Kick-Brauckman's pots of $10 \mathrm{dm}^{3}$ capacity. After germination, the number of plants was reduced to 10 . The pots were filled with a mixture of sandy loam and sand $(7: 2, w / w)$. The analysis of the water retention curve of this substrate showed that a $\mathrm{pF}$ range of 2.2-3.0 indicate easily available water. At the $\mathrm{pF}$ 3.0-4.2, water was less available, creating the conditions of drought stress, and $\mathrm{pF}>4.2$ was a permanent wilting point [19]. In the present study, two water regimes were used: control conditions with the $\mathrm{pF}$ range of $\mathrm{pF}$ 2.2-3.0 and a mild drought with $\mathrm{pF}$ value kept at the range of 3.4-3.6, which was applied at the 3-leaf seedling stage and lasted for 10 days. The experiment was started in spring (April), and plants were grown in a glasshouse with natural light conditions. The soil moisture was controlled daily using an FOM/mts TDR soil moisture meter according to the reflectometry method (Easy Test, Institute of Agrophysics PAS, Lublin, Poland) and adjusted by adding the appropriate quantity of water. The experiment was carried out in three replicates and tissue for RNA extraction was collected from five plants per replicate.

\subsection{RNA Isolation}

After 10 days of drought stress, the second leaf and the whole root system were collected for RNA extraction from both drought-treated and control plants. To collect roots, plants were gently removed from the soil, separated from each other and roots were briefly washed in water to remove the soil substrate. The washing time did not exceed $30 \mathrm{~s}$. Leaves and roots were frozen in liquid nitrogen and were subsequently homogenized in a sterile, ice-cold mortar. Total RNA was extracted using an RNeasy Plant Mini kit (Qiagen, Hilden, Germany), according to the manufacturer's instructions. 
Extracted RNA was additionally purified using precipitation in $1 \mathrm{M}$ lithium chloride, and each RNA precipitate was then dissolved in $15 \mu \mathrm{L}$ of nuclease-free $\mathrm{H}_{2} \mathrm{O}$. The yield and purity of the RNA were determined using a NanoDrop ND-1000 spectrophotometer (NanoDrop Technologies, Wilmington, DE, USA). The integrity of the RNA was checked using denaturation agarose gel electrophoresis using pre-cast gels and FlashGel RNA System (Lonza, Basel, Switzerland).

\subsection{Preparation of Microarrays and Microarray Data Analysis}

The synthesis, labeling, and hybridization of cDNA and cRNA to $4 \times 44 \mathrm{~K}$ Agilent Barley gene expression arrays (Agilent Technologies, Santa Clara, CA, USA) were carried out at the Genomics Core Facility, European Molecular Biology Laboratory (EMBL), Heidelberg, Germany, as described earlier in Kwasniewski et al. [66]. One technical replication of microarray hybridization was made for each of the biological replications representing different experimental combinations of genotype, organs, and the type of treatment. The microarray data were analyzed using GeneSpring GX 12.5 software (Agilent Technologies). Hybridization data were subjected to per chip normalization using the percentile shift method to the 75th percentile. A baseline transformation was then performed to the median of all of the samples. Statistical testing for differential expression was performed using two-way ANOVA followed by the Benjamini-Hochberg false discovery rate (FDR) correction for multiple testing [67]. Fold change (FC) $\geq 3$ ( $p \leq 0.05$ after FDR correction) was considered as a differential expression of a gene between drought-treated and control samples. Raw microarray data, normalized intensity values, and corresponding metadata are accessible through the Gene Expression Omnibus (GEO) repository under the accession number GSE128048.

\subsection{Agilent Barley Gene Expression Microarray Annotation, GO Enrichment and Transcription Factor Encoding Genes Analysis}

Blast2Go software (BioBam Bioinformatics S.L, Valencia, Spain) [68] and the blastn algorithm were used to map probes sequences from the Agilent Barley gene expression microarray to cDNA sequences representing high-confidence $(\mathrm{HC})$ barley genes which were retrieved from the Ensembl Plants database (http://plants.ensembl.org), using the barley genome assembly version Hv_IBSC_PGSB_v2. The threshold values for the blastn settings were as follows: E-value- $1.03 \times 10^{-3}$, word size -9 , and high-scoring segment pair (HSP) - 33, with low complexity filter applied. The functional annotations of HC genes was used after Mascher et al. [15]. Because several online databases still utilize the data from the first version of barley genome assembly, similar annotation of microarray probes was done using the old version of the genome (version 082214v1, EnsemblPlants database). For this reason, all results in supplementary materials and, when applicable, in the main text, are presented using two gene identifiers abbreviated as HORVU (for the new genome assembly) and MLOC (for the old assembly).

The functional characterization of all identified DEGs was performed using the KEGG database. To assign DEGs into KEGG orthologous groups, a BlastKOALA tool was applied [69], and the KEGG identifiers were then used to reconstruct KEGG pathways and BRITE hierarchies available at KEGG service (https://www.genome.jp/kegg/kegg2.html). Gene ontology enrichment analysis was carried out separately for up- and down-regulated root DEGs using singular enrichment analysis (SEA) available through the AgriGO v2.0 toolkit (http://systemsbiology.cau.edu.cn/agriGOv2). Here, a hypergeometric statistical test, at a significance level of $p=0.01$ and minimum mapping entries of 5 , was used. The GO data for the whole set of barley genes from the IPK Barley BLAST server were taken as a background reference for the analysis. The individual enrichment results were then compared using the SEACOMPARE tool from AgriGO v2.0. The GO slim analysis was done using a GOSlim viewer provided by AgBase (http://agbase.arizona.edu/cgi-bin/tools).

In order to find genes encoding transcription factors (TFs) and other gene expression regulatory proteins, the protein sequences for all root DEGs were retrieved from the EnsemblPlant database (version Hv_IBSC_PGSB_v2) and analyzed using the iTAK (plant transcription factor and protein 
kinase identifier and classifier) program [70]. The analysis of TFs with significantly enriched putative targets was performed using the TF enrichment tool from the Plant Transcriptional Regulatory Map (http://plantregmap.cbi.pku.edu.cn/tf_enrichment.php) and a "motif" method with threshold $p$-value $\leq 0.05$ was applied to find possible regulatory interactions between TFs and their putative targets. All Venn diagrams were drawn using Venny 2.1 [71].

\subsection{Quantitative Reverse Transcription (RT)-qPCR}

One microgram of total RNA was subjected to DNase treatment and subsequent cDNA synthesis using RevertAid First Strand cDNA Synthesis Kit (Thermo Fisher Scientific, Waltham, MA USA) according to the manufacturer's instructions. The cDNA was diluted 1:5 with $\mathrm{ddH}_{2} \mathrm{O}$ and used as a template for the qPCR. The primers that were used in the qPCR were designed using Quant-Prime software (http://www.quantprime.de). The $10 \mu \mathrm{L}$ qPCR reaction contained $2 \mu \mathrm{L}$ of cDNA, $1 \mu \mathrm{L}$ of the primer pair mixture $(5 \mu \mathrm{M})$ ), and $5 \mu \mathrm{L}$ of $2 \times$ Master Mix (LightCycler 480 SYBR Green I Master; Roche, Indianapolis, Indiana, USA). The qPCR protocol for the amplification on LightCycler 480 real-time PCR instrument (Roche) using the SYBR Green I method was as follows: initial denaturation for $10 \mathrm{~min}$ at $95^{\circ} \mathrm{C}$, followed by 45 cycles of $10 \mathrm{~s}$ at $95^{\circ} \mathrm{C}, 15 \mathrm{~s}$ at $60^{\circ} \mathrm{C}$ and $10 \mathrm{~s}$ at $72{ }^{\circ} \mathrm{C}$, followed by a melting-curve analysis. The gene for ADP-ribosylation factor 1 was used as a reference [72]. All analyses were done in three biological replicates. Amplification efficiencies were calculated using LinRegPCR [73]. Calculations of the fold change of expression (FC) were made using the Pfaffl method [74]. Statistical significance of expression differences between control and drought treated samples were tested using REST software [75]. Spearman's rank correlation coefficient was used to statistically compare microarray and qPCR FC data.

Supplementary Materials: Supplementary materials can be found at http://www.mdpi.com/1422-0067/20/24/ 6139/s1.

Author Contributions: Conceptualization, A.J. and I.S.; Formal analysis, A.J.; Funding acquisition, A.J. and I.S.; Investigation, A.J., M.K., M.S., A.K., K.M. and P.O.; Methodology, A.J., I.S. and A.K.; Supervision, A.J.; Writing—original draft, A.J.; Writing-review \& editing, I.S.

Funding: This work was supported by the European Regional Development Fund through the Innovative Economy Program for Poland 2007-2013, project WND-POIG.01.03.01-00-101/08 POLAPGEN-BD “Biotechnological tools for breeding cereals with increased resistance to drought". All information about the project can be found at www.polapgen.pl.

Acknowledgments: We greatly acknowledge Vladimir Benes and Paul Collier (Genomics Core Facility at EMBL, Heidelberg) for performing probe synthesis and microarray hybridizations and Grzegorz Józefaciuk (Institute of Agrophysics of the Polish Academy of Sciences, Lublin, Poland) for physical characterization of soil substrate by water retention curve $\mathrm{pF}$ analysis.

Conflicts of Interest: The authors declare no conflict of interest.

\section{Abbreviations}

DEG Differentially expressed gene

GO Gene ontology

PSI Photosystem I

PSII Photosystem II

ROS Reactive oxygen species

TF Transcription factor

\section{References}

1. Schachtman, D.P.; Goodger, J.Q.D. Chemical root to shoot signaling under drought. Trends Plant Sci. 2008, 13, 281-287. [CrossRef] [PubMed]

2. Comas, L.H.; Becker, S.R.; Cruz, V.M.V.; Byrne, P.F.; Dierig, D.A. Root traits contributing to plant productivity under drought. Front. Plant Sci. 2013, 4, 442. [CrossRef] [PubMed] 
3. Hu, L.; Xie, Y.; Fan, S.; Wang, Z.; Wang, F.; Zhang, B.; Li, H.; Song, J.; Kong, L. Comparative analysis of root transcriptome profiles between drought-tolerant and susceptible wheat genotypes in response to water stress. Plant Sci. 2018, 272, 276-293. [CrossRef] [PubMed]

4. Zhang, C.; Zhang, L.; Zhang, S.; Zhu, S.; Wu, P.; Chen, Y.; Li, M.; Jiang, H.; Wu, G. Global analysis of gene expression profiles in physic nut (Jatropha curcas L.) seedlings exposed to drought stress. BMC Plant Biol. 2015, 15, 17. [CrossRef]

5. Sahoo, K.K.; Tripathi, A.K.; Pareek, A.; Singla-Pareek, S.L. Taming drought stress in rice through genetic engineering and transcription factors and protein kinases. Plant Stress 2013, 7, 60-72.

6. Janiak, A.; Kwaśniewski, M.; Szarejko, I. Gene expression regulation in roots under drought. J. Exp. Bot. 2016, 67, 1003-1014. [CrossRef]

7. Tripathi, P.; Rabara, R.C.; Rushton, P.J. A systems biology perspective on the role of WRKY transcription factors in drought responses in plants. Planta 2014, 239, 255-266. [CrossRef]

8. Mun, B.-G.; Lee, S.-U.; Park, E.-J.; Kim, H.-H.; Hussain, A.; Imran, Q.M.; Lee, I.-J.; Yun, B.-W. Analysis of transcription factors among differentially expressed genes induced by drought stress in Populus davidiana. 3 Biotech 2017, 7, 209. [CrossRef]

9. Wu, J.; Wang, L.; Wang, S. Comprehensive analysis and discovery of drought-related NAC transcription factors in common bean. BMC Plant Biol. 2016, 16, 193. [CrossRef]

10. Agarwal, P.; Agarwal, P.K.; Joshi, A.J.; Sopory, S.K.; Reddy, M.K. Overexpression of PgDREB2A transcription factor enhances abiotic stress tolerance and activates downstream stress-responsive genes. Mol. Biol. Rep. 2010, 37, 1125-1135. [CrossRef]

11. Jung, H.; Chung, P.J.; Park, S.-H.; Redillas, M.C.F.R.; Kim, Y.S.; Suh, J.-W.; Kim, J.-K. Overexpression of OsERF48 causes regulation of OsCML16, a calmodulin-like protein gene that enhances root growth and drought tolerance. Plant Biotechnol. J. 2017, 15, 1295-1308. [CrossRef]

12. Jeong, J.S.; Kim, Y.S.; Redillas, M.C.F.R.; Jang, G.; Jung, H.; Bang, S.W.; Choi, Y.D.; Ha, S.-H.; Reuzeau, C.; Kim, J.-K. OsNAC5 overexpression enlarges root diameter in rice plants leading to enhanced drought tolerance and increased grain yield in the field. Plant Biotechnol. J. 2013, 11, 101-114. [CrossRef]

13. Lee, D.-K.; Jung, H.; Jang, G.; Jeong, J.S.; Kim, Y.S.; Ha, S.-H.; Do Choi, Y.; Kim, J.-K. Overexpression of the OsERF71 transcription factor alters rice root structure and drought resistance. Plant Physiol. 2016, 172, 575-588. [CrossRef]

14. Forti, R. (Ed.) Eurostat Report: Agriculture, Forestry and Fishery Statistics; Publications Office of the European Union: Luxembourg, 2017.

15. Mascher, M.; Gundlach, H.; Himmelbach, A.; Beier, S.; Twardziok, S.O.; Wicker, T.; Radchuk, V.; Dockter, C.; Hedley, P.E.; Russell, J.; et al. A chromosome conformation capture ordered sequence of the barley genome. Nature 2017, 544, 427-433. [CrossRef]

16. Filek, M.; Łabanowska, M.; Kościelniak, J.; Biesaga-Kościelniak, J.; Kurdziel, M.; Szarejko, I.; Hartikainen, H. Characterization of barley leaf tolerance to drought stress by chlorophyll fluorescence and electron paramagnetic resonance studies. J. Agron. Crop Sci. 2015, 201, 228-240. [CrossRef]

17. Filek, M.; Łabanowska, M.; Kurdziel, M.; Wesełucha-Birczyńska, A.; Bednarska-Kozakiewicz, E. Structural and biochemical response of chloroplasts in tolerant and sensitive barley genotypes to drought stress. J. Plant Physiol. 2016, 207, 61-72. [CrossRef]

18. Chmielewska, K.; Rodziewicz, P.; Swarcewicz, B.; Sawikowska, A.; Krajewski, P.; Marczak, Ł.; Ciesiołka, D.; Kuczyńska, A.; Mikołajczak, K.; Ogrodowicz, P.; et al. Analysis of drought-induced proteomic and metabolomic changes in barley (Hordeum vulgare L.) leaves and roots unravels some aspects of biochemical mechanisms involved in drought tolerance. Front. Plant Sci. 2016, 7, 1108. [CrossRef]

19. Mikołajczak, K.; Kuczyńska, A.; Krajewski, P.; Sawikowska, A.; Surma, M.; Ogrodowicz, P.; Adamski, T.; Krystkowiak, K.; Górny, A.G.; Kempa, M.; et al. Quantitative trait loci for plant height in Maresi $\times$ CamB barley population and their associations with yield-related traits under different water regimes. J. Appl. Genet. 2017, 58, 23-35. [CrossRef]

20. Janiak, A.; Kwasniewski, M.; Sowa, M.; Gajek, K.; Żmuda, K.; Kościelniak, J.; Szarejko, I. No time to waste: Transcriptome study reveals that drought tolerance in barley may be attributed to stressed-like expression patterns that exist before the occurrence of stress. Front. Plant Sci. 2017, 8, 2212. [CrossRef]

21. Olesen, C.; Møller, M.; Byskov, A.G. Tesmin transcription is regulated differently during male and female meiosis. Mol. Reprod. Dev. 2004, 67, 116-126. [CrossRef] 
22. Andersen, S.U.; Algreen-Petersen, R.G.; Hoedl, M.; Jurkiewicz, A.; Cvitanich, C.; Braunschweig, U.; Schauser, L.; Oh, S.-A.; Twell, D.; Jensen, E.Ø. The conserved cysteine-rich domain of a tesmin/TSO1-like protein binds zinc in vitro and TSO1 is required for both male and female fertility in Arabidopsis thaliana. J. Exp. Bot. 2007, 58, 3657-3670. [CrossRef] [PubMed]

23. Wang, W.; Sijacic, P.; Xu, P.; Lian, H.; Liu, Z. Arabidopsis TSO1 and MYB3R1 form a regulatory module to coordinate cell proliferation with differentiation in shoot and root. Proc. Natl. Acad. Sci. USA 2018, 115, E3045-E3054. [CrossRef] [PubMed]

24. Maeda, Y.; Konishi, M.; Kiba, T.; Sakuraba, Y.; Sawaki, N.; Kurai, T.; Ueda, Y.; Sakakibara, H.; Yanagisawa, S. A NIGT1-centred transcriptional cascade regulates nitrate signalling and incorporates phosphorus starvation signals in Arabidopsis. Nat. Commun. 2018, 9, 1376. [CrossRef] [PubMed]

25. Kiba, T.; Inaba, J.; Kudo, T.; Ueda, N.; Konishi, M.; Mitsuda, N.; Takiguchi, Y.; Kondou, Y.; Yoshizumi, T.; Ohme-Takagi, M.; et al. Repression of nitrogen starvation responses by members of the arabidopsis GARP-Type transcription factor NIGT1/HRS1 subfamily. Plant Cell 2018, 30, 925-945. [CrossRef] [PubMed]

26. Ruan, W.; Guo, M.; Wu, P.; Yi, K. Phosphate starvation induced OsPHR4 mediates Pi-signaling and homeostasis in rice. Plant Mol. Biol. 2017, 93, 327-340. [CrossRef] [PubMed]

27. López-Bucio, J.; Cruz-Ramírez, A.; Herrera-Estrella, L. The role of nutrient availability in regulating root architecture. Curr. Opin. Plant Biol. 2003, 6, 280-287. [CrossRef]

28. Deak, K.I.; Malamy, J. Osmotic regulation of root system architecture. Plant J. 2005, 43, 17-28. [CrossRef]

29. Brandt, D.T.; Baarlink, C.; Kitzing, T.M.; Kremmer, E.; Ivaska, J.; Nollau, P.; Grosse, R. SCAI acts as a suppressor of cancer cell invasion through the transcriptional control of beta1-integrin. Nat. Cell Biol. 2009, 11, 557-568. [CrossRef]

30. Brauchle, M.; Yao, Z.; Arora, R.; Thigale, S.; Clay, I.; Inverardi, B.; Fletcher, J.; Taslimi, P.; Acker, M.G.; Gerrits, B.; et al. Protein complex interactor analysis and differential activity of KDM3 subfamily members towards H3K9 methylation. PLoS ONE 2013, 8, e60549. [CrossRef]

31. Kreßner, C.; Nollau, P.; Grosse, R.; Brandt, D.T. Functional interaction of SCAI with the SWI/SNF complex for transcription and tumor cell invasion. PLoS ONE 2013, 8, e69947. [CrossRef]

32. Yang, N.; Xu, R.-M. Structure and function of the BAH domain in chromatin biology. Crit. Rev. Biochem. Mol. Biol. 2013, 48, 211-221. [CrossRef] [PubMed]

33. Zemach, A.; Grafi, G. Methyl-CpG-binding domain proteins in plants: Interpreters of DNA methylation. Trends Plant Sci. 2007, 12, 80-85. [CrossRef] [PubMed]

34. Licausi, F.; Ohme-Takagi, M.; Perata, P. APETALA2/Ethylene Responsive Factor (AP2/ERF) transcription factors: Mediators of stress responses and developmental programs. New Phytol. 2013, 199, 639-649. [CrossRef] [PubMed]

35. Lata, C.; Mishra, A.K.; Muthamilarasan, M.; Bonthala, V.S.; Khan, Y.; Prasad, M. Genome-wide investigation and expression profiling of AP2/ERF transcription factor superfamily in foxtail millet (Setaria italica L.). PLoS ONE 2014, 9, e113092. [CrossRef]

36. Fernández-Calvo, P.; Chini, A.; Fernández-Barbero, G.; Chico, J.-M.; Gimenez-Ibanez, S.; Geerinck, J.; Eeckhout, D.; Schweizer, F.; Godoy, M.; Franco-Zorrilla, J.M.; et al. The Arabidopsis bHLH transcription factors MYC3 and MYC4 are targets of JAZ repressors and act additively with MYC2 in the activation of jasmonate responses. Plant Cell 2011, 23, 701-715. [CrossRef]

37. Ye, H.; Du, H.; Tang, N.; Li, X.; Xiong, L. Identification and expression profiling analysis of TIFY family genes involved in stress and phytohormone responses in rice. Plant Mol. Biol. 2009, 71, 291-305. [CrossRef]

38. Gao, Q.-M.; Venugopal, S.; Navarre, D.; Kachroo, A. Low oleic acid-derived repression of jasmonic acid-inducible defense responses requires the WRKY50 and WRKY51 proteins. Plant Physiol. 2011, 155, 464-476. [CrossRef]

39. Pan, L.; Zhang, X.; Wang, J.; Ma, X.; Zhou, M.; Huang, L.; Nie, G.; Wang, P.; Yang, Z.; Li, J. Transcriptional profiles of drought-related genes in modulating metabolic processes and antioxidant defenses in Lolium multiflorum. Front. Plant Sci. 2016, 7, 519. [CrossRef]

40. Srinivasan, T.; Kumar, K.R.R.; Meur, G.; Kirti, P.B. Heterologous expression of Arabidopsis NPR1 (AtNPR1) enhances oxidative stress tolerance in transgenic tobacco plants. Biotechnol. Lett. 2009, 31, 1343-1351. [CrossRef]

41. Bolduc, N.; Hake, S. The maize transcription factor KNOTTED1 directly regulates the gibberellin catabolism gene ga2ox1. Plant Cell 2009, 21, 1647-1658. [CrossRef] 
42. Ubeda-Tomás, S.; Federici, F.; Casimiro, I.; Beemster, G.T.S.; Bhalerao, R.; Swarup, R.; Doerner, P.; Haseloff, J.; Bennett, M.J. Gibberellin signaling in the endodermis controls Arabidopsis root meristem size. Curr. Biol. 2009, 19, 1194-1199. [CrossRef]

43. Huang, H.; Liu, B.; Liu, L.; Song, S. Jasmonate action in plant growth and development. J. Exp. Bot. 2017, 68, 1349-1359. [CrossRef]

44. Wang, L.; Zhu, J.; Li, X.; Wang, S.; Wu, J. Salt and drought stress and ABA responses related to $b$ ZIP genes from V. radiata and V. angularis. Gene 2018, 651, 152-160. [CrossRef] [PubMed]

45. Nijhawan, A.; Jain, M.; Tyagi, A.K.; Khurana, J.P. Genomic survey and gene expression analysis of the basic leucine zipper transcription factor family in rice. Plant Physiol. 2008, 146, 333-350. [CrossRef] [PubMed]

46. Shaikhali, J.; Norén, L.; de Dios Barajas-López, J.; Srivastava, V.; König, J.; Sauer, U.H.; Wingsle, G.; Dietz, K.-J.; Strand, Å. Redox-mediated mechanisms regulate DNA binding activity of the G-group of basic region leucine zipper (bZIP) transcription factors in Arabidopsis. J. Biol. Chem. 2012, 287, 27510-27525. [CrossRef] [PubMed]

47. Ikeda, M.; Mitsuda, N.; Ohme-Takagi, M. Arabidopsis HsfB1 and HsfB2b act as repressors of the expression of heat-inducible Hsfs but positively regulate the acquired thermotolerance. Plant Physiol. 2011, 157, 1243-1254. [CrossRef]

48. Chiu, R.S.; Nahal, H.; Provart, N.J.; Gazzarrini, S. The role of the Arabidopsis FUSCA3 transcription factor during inhibition of seed germination at high temperature. BMC Plant Biol. 2012, 12, 15. [CrossRef]

49. Zhang, X.; Liu, X.; Zhang, D.; Tang, H.; Sun, B.; Li, C.; Hao, L.; Liu, C.; Li, Y.; Shi, Y.; et al. Genome-wide identification of gene expression in contrasting maize inbred lines under field drought conditions reveals the significance of transcription factors in drought tolerance. PLOS ONE 2017, 12, e0179477. [CrossRef]

50. Seo, P.J.; Park, C.-M. Auxin homeostasis during lateral root development under drought condition. Plant Signal. Behav. 2009, 4, 1002-1004. [CrossRef]

51. Hirsch, S.; Oldroyd, G.E.D. GRAS-domain transcription factors that regulate plant development. Plant Signal. Behav. 2009, 4, 698-700. [CrossRef]

52. Chung, H.S.; Howe, G.A. A critical role for the TIFY motif in repression of jasmonate signaling by a stabilized splice variant of the JASMONATE ZIM-domain protein JAZ10 in Arabidopsis. Plant Cell 2009, 21, 131-145. [CrossRef] [PubMed]

53. Aharoni, A.; Dixit, S.; Jetter, R.; Thoenes, E.; van Arkel, G.; Pereira, A. The SHINE clade of AP2 domain transcription factors activates wax biosynthesis, alters cuticle properties, and confers drought tolerance when overexpressed in Arabidopsis. Plant Cell 2004, 16, 2463-2480. [CrossRef] [PubMed]

54. Shi, J.X.; Malitsky, S.; De Oliveira, S.; Branigan, C.; Franke, R.B.; Schreiber, L.; Aharoni, A. SHINE transcription factors act redundantly to pattern the archetypal surface of Arabidopsis flower organs. PLoS Genet. 2011, 7, e1001388. [CrossRef] [PubMed]

55. Kreszies, T.; Schreiber, L.; Ranathunge, K. Suberized transport barriers in Arabidopsis, barley and rice roots: From the model plant to crop species. J. Plant Physiol. 2018, 227, 75-83. [CrossRef]

56. Baxter, I.; Hosmani, P.S.; Rus, A.; Lahner, B.; Borevitz, J.O.; Muthukumar, B.; Mickelbart, M.V.; Schreiber, L.; Franke, R.B.; Salt, D.E. Root suberin forms an extracellular barrier that affects water relations and mineral nutrition in Arabidopsis. PLoS Genet. 2009, 5, e1000492. [CrossRef]

57. Shuai, B.; Reynaga-Pena, C.G.; Springer, P.S. The LATERAL ORGAN BOUNDARIES gene defines a novel, plant-specific gene family. Plant Physiol. 2002, 129, 747-761. [CrossRef]

58. Ariel, F.D.; Diet, A.; Crespi, M.; Chan, R.L. The LOB-like transcription factor MtLBD1 controls Medicago truncatula root architecture under salt stress. Plant Signal. Behav. 2010, 5, 1666-1668. [CrossRef]

59. Xu, C.; Tai, H.; Saleem, M.; Ludwig, Y.; Majer, C.; Berendzen, K.W.; Nagel, K.A.; Wojciechowski, T.; Meeley, R.B.; Taramino, G.; et al. Cooperative action of the paralogous maize lateral organ boundaries (LOB) domain proteins RTCS and RTCL in shoot-borne root formation. New Phytol. 2015, 207, 1123-1133. [CrossRef]

60. Taramino, G.; Sauer, M.; Stauffer, J.L.; Multani, D.; Niu, X.; Sakai, H.; Hochholdinger, F. The maize (Zea mays L.) RTCS gene encodes a LOB domain protein that is a key regulator of embryonic seminal and post-embryonic shoot-borne root initiation. Plant J. 2007, 50, 649-659. [CrossRef]

61. Liu, H.; Wang, S.; Yu, X.; Yu, J.; He, X.; Zhang, S.; Shou, H.; Wu, P. ARL1, a LOB-domain protein required for adventitious root formation in rice. Plant J. 2005, 43, 47-56. [CrossRef] 
62. Ranjan, A.; Sawant, S. Genome-wide transcriptomic comparison of cotton (Gossypium herbaceum) leaf and root under drought stress. 3 Biotech 2015, 5, 585-596. [CrossRef] [PubMed]

63. Molina, C.; Rotter, B.; Horres, R.; Udupa, S.M.; Besser, B.; Bellarmino, L.; Baum, M.; Matsumura, H.; Terauchi, R.; Kahl, G.; et al. SuperSAGE: The drought stress-responsive transcriptome of chickpea roots. BMC Genom. 2008, 9, 553. [CrossRef] [PubMed]

64. Cohen, D.; Bogeat-Triboulot, M.-B.; Tisserant, E.; Balzergue, S.; Martin-Magniette, M.-L.; Lelandais, G.; Ningre, N.; Renou, J.-P.; Tamby, J.-P.; Le Thiec, D.; et al. Comparative transcriptomics of drought responses in Populus: A meta-analysis of genome-wide expression profiling in mature leaves and root apices across two genotypes. BMC Genom. 2010, 11, 630. [CrossRef] [PubMed]

65. Lorenz, W.W.; Alba, R.; Yu, Y.-S.; Bordeaux, J.M.; Simões, M.; Dean, J.F.D. Microarray analysis and scale-free gene networks identify candidate regulators in drought-stressed roots of loblolly pine (P. taeda L.). BMC Genom. 2011, 12, 264. [CrossRef]

66. Kwasniewski, M.; Daszkowska-Golec, A.; Janiak, A.; Chwialkowska, K.; Nowakowska, U.; Sablok, G.; Szarejko, I. Transcriptome analysis reveals the role of the root hairs as environmental sensors to maintain plant functions under water-deficiency conditions. J. Exp. Bot. 2016, 67, 1079-1094. [CrossRef]

67. Benjamini, Y.; Hochberg, Y. Controlling the false discovery rate: A practical and powerful approach to multiple testing. J. R. Stat. Soc. B Met. 1995, 57, 289-300. [CrossRef]

68. Götz, S.; García-Gómez, J.M.; Terol, J.; Williams, T.D.; Nagaraj, S.H.; Nueda, M.J.; Robles, M.; Talón, M.; Dopazo, J.; Conesa, A. High-throughput functional annotation and data mining with the Blast2GO suite. Nucleic Acids Res. 2008, 36, 3420-3435. [CrossRef]

69. Kanehisa, M.; Sato, Y.; Morishima, K. BlastKOALA and GhostKOALA: KEGG tools for functional characterization of genome and metagenome sequences. J. Mol. Biol. 2016, 428, 726-731. [CrossRef] [PubMed]

70. Zheng, Y.; Jiao, C.; Sun, H.; Rosli, H.G.; Pombo, M.A.; Zhang, P.; Banf, M.; Dai, X.; Martin, G.B.; Giovannoni, J.J.; et al. ITAK: A program for genome-wide prediction and classification of plant transcription factors, transcriptional regulators, and protein kinases. Mol. Plant 2016, 9, 1667-1670. [CrossRef]

71. Oliveros, J.C. Venny. An interactive tool for comparing lists with Venn's diagrams. Available online: http://bioinfogp.cnb.csic.es/tools/venny/index.html (accessed on 4 December 2019).

72. Rapacz, M.; Stẹpień, A.; Skorupa, A. Internal standards for quantitative RT-PCR studies of gene expression under drought treatment in barley (Hordeum vulgare L.): The effects of developmental stage and leaf age. Acta Phys. Plant. 2012, 34, 1723-1733. [CrossRef]

73. Ramakers, C.; Ruijter, J.M.; Deprez, R.H.L.; Moorman, A.F.M. Assumption-free analysis of quantitative real-time polymerase chain reaction (PCR) data. Neurosci. Lett. 2003, 339, 62-66. [CrossRef]

74. Pfaffl, M.W. A new mathematical model for relative quantification in real-time RT-PCR. Nucleic Acids Res. 2001, 29, e45. [CrossRef]

75. Pfaffl, M.W.; Horgan, G.W.; Dempfle, L. Relative expression software tool (RESTC) for group-wise comparison and statistical analysis of relative expression results in real-time PCR. Nucleic Acids Res. 2002, 30 , e36. [CrossRef] [PubMed]

(C) 2019 by the authors. Licensee MDPI, Basel, Switzerland. This article is an open access article distributed under the terms and conditions of the Creative Commons Attribution (CC BY) license (http://creativecommons.org/licenses/by/4.0/). 